Copyright

by

Maximilian Michael Schadegg

2013 
The Report Committee for Maximilian Michael Schadegg Certifies that this is the approved version of the following report:

\title{
Jovian Orbit Capture and Eccentricity Reduction Using Electrodynamic Tether Propulsion
}

\author{
APPROVED BY
}

SUPERVISING COMMITTEE:

Supervisor:

Ryan P. Russell

Gregory Lantoine 


\title{
Jovian Orbit Capture and Eccentricity Reduction Using Electrodynamic Tether Propulsion
}

\author{
by
}

Maximilian Michael Schadegg, B.S.

\section{REPORT}

Presented to the Faculty of the Graduate School of

The University of Texas at Austin

in Partial Fulfillment

of the Requirements

for the Degree of

MASTER OF SCIENCE IN ENGINEERING

THE UNIVERSITY OF TEXAS AT AUSTIN

December 2013 
To my family and friends past, present, and future. 


\section{Acknowledgments}

This work was made possible by the support of several individuals and groups.

I would foremost like to thank my advisor, Ryan Russell, for guiding me through my graduate education and research. I would also like to thank my committee member and principal investigator on this project, Gregory Lantoine, and all those other members involved on the project at the Jet Propulsion Laboratory.

This work was supported by a NASA Innovative Advanced Concepts (NIAC) Phase I contract in partnership with NASA JPL. Financial support to present this work was also provided by an award from the American Astronautical Society. I would lastly like to thank my colleagues and friends, especially Etienne Pellegrini, Vivek Vittaldev, Denis Felikson, Kevin Bokelmann, Ashley Biria and Demyan Lantukh, for helping and putting up with me along the way. 


\title{
Jovian Orbit Capture and Eccentricity Reduction Using Electrodynamic Tether Propulsion
}

\author{
by \\ Maximilian Michael Schadegg, M.S.E. \\ The University of Texas at Austin, 2013 \\ SUPERVISOR: Ryan P. Russell
}

The use of electrodynamic tethers for propulsion and power generation is attractive for missions to the outer planets, which are traditionally handicapped by large propellant requirements, large times of flight, and a scarcity of power available. The proposed electrodynamic tether propulsion scheme is shown to be capable at reducing or eliminating these mission constraints. In this work, the orbital dynamics of a spacecraft using electrodynamic tether propulsion during the mission phases of capture, apojove pump-down and perijove pumpup in the Jovian system are investigated.

The main result is the mapped design space involving mission duration, tether length and minimum perijove radius. Phase-free flyby sequences and bang-bang control laws are also included, which provide performance upper bounds for a given mission architecture. It is found to be advantageous to utilize in-bound only flybys of the Galilean moons during capture, and few, if any, out-bound only flybys during apojove pump-down. The electrodynamic tether system is also shown to be capable of lowering the spacecraft's orbit 
to a Europa-Ganymede Hohmann orbit with a total flight time after entering Jupiter's sphere of influence of just under two years. The benefits of leveraging solar third body perturbations, ballistic flyby tours, and adding a secondary propulsion system are also considered. 


\section{Table of Contents}

Acknowledgments $\quad$ v

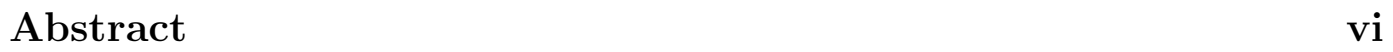

List of Tables $\quad$ x

List of Figures $\quad$ xi

$\begin{array}{lll}\text { Chapter 1. Introduction } & 1\end{array}$

Chapter 2. Physics of Electrodynamic Tethers 4

$\begin{array}{llr}\text { Chapter 3. } & \text { Mission Architecture } & 8\end{array}$

3.1 Mission Phases . . . . . . . . . . . . . . 8

3.2 Assumptions . . . . . . . . . . . . . . . . . . 10

Chapter 4. Capture and Apojove Pump-Down 12

4.1 Capture Without Flybys . . . . . . . . . . . . . . . 14

4.1 .1 Design Space . . . . . . . . . . . . . . . . . . . 14

4.1.2 Baseline Trajectories . . . . . . . . . . . . . . 17

4.2 Capture With Flybys . . . . . . . . . . . . . . . . . . 20

4.2.1 In-Bound Flybys . . . . . . . . . . . . . . . . . . 20

4.2 .2 Out-Bound Flybys . . . . . . . . . . . . . . . . 22

4.2.3 Out-Bound Flybys with Constant Perijove . . . . . . . . 23

$\begin{array}{lll}\text { Chapter 5. Perijove Pump-Up } & 29\end{array}$

5.1 Tether Control Law . . . . . . . . . . . . . . . . . . . . . . . . 29

5.2 Third Body Perturbations . . . . . . . . . . . . . . 33

5.3 Ballistic Flybys . . . . . . . . . . . . . . . . . . . 38 
Chapter 6. Conclusions and Future Work 40

6.1 Summary. . . . . . . . . . . . . . . . . . 40

6.2 Future Work . . . . . . . . . . . . . . . . . . . 41

$\begin{array}{ll}\text { Bibliography } & 43\end{array}$ 


\section{List of Tables}

4.1 The required $\Delta \mathrm{V}$ burns applied at apojove to keep a constant perijove of $1.5 \mathrm{R}_{J}$ for each revolution. . . . . . . . . .

5.1 Maximum perijove radius achieved after 1 year on $\left[K_{1}, K_{2}\right]$, starting with apojove radius at each Jovian moon. . . . . . .

33 


\section{List of Figures}

2.1 Lorentz force generation diagram of conducting wire passing through a planet's magnetic field. . . . . . . . . . . . .

2.2 Lorentz force generated versus semi-major axis of a circular orbit about Jupiter. . . . . . . . . . . . . . . .

3.1 Phases of MAGNETOUR mission concept [1] from left to right: capture; apojove pump-down; perijove pump-up; and loosely captured moon tour. . . . . . . . . . . . . . . . .

3.2 Mock-up spacecraft model from the MAGNETOUR mission concept [1], which shows the tether system configuration. . . .

4.1 Capture orbit evolutions with a $50 \mathrm{~km}$ tether for varying perijove radii. . . . . . . . . . . . . . . . . .

4.2 The maximum Lorentz force generated during insertion (left) and the corresponding total $\Delta \mathrm{V}$ change (right) for varying perijove radii and tether lengths. . . . . . . . . . . . .

4.3 The total time needed to capture and lower apojove radius to Callisto for varying perijove radii and tether lengths. . . . . .

4.4 The maximum temperature on the tether during insertion for varying perijove radii and tether lengths. . . . . . . . . .

4.5 Characteristic trajectory for a $50 \mathrm{~km}$ tether spacecraft with a perijove radius of $2.0 \mathrm{R}_{J} \ldots \ldots \ldots \ldots$. . . . . . .

4.6 Normal (left) and magnified (right) views of the Lorentz force versus time for a $50 \mathrm{~km}$ tether with a periojve radius of $2.0 \mathrm{R}_{J}$.

4.7 Characteristic trajectory for a $20 \mathrm{~km}$ tether spacecraft with a perijove radius of $1.5 \mathrm{R}_{J} \ldots \ldots \ldots$. . . . . . .

4.8 Normal (left) and magnified (right) views of the Lorentz force versus time for a $20 \mathrm{~km}$ tether with a periojve radius of $1.5 \mathrm{R}_{J}$.

4.9 Vector diagram showing $\mathrm{V}_{\infty}$ and spacecraft velocity vectors before the flyby and the corresponding vectors after the flyby (not to scale). . . . . . . . . . . . . . .

4.10 The position evolution of a spacecraft with no flybys (blue) and with flybys of Callisto, Ganymede, Europa and Io (green). . 
4.11 Normal (left) and magnified (right) plots of energy versus time of a spacecraft with no flybys (blue) and with flybys of Callisto, Ganymede, Europa and Io (green). . . . . . . . . . . . . . .

4.12 Normal (left) and magnified (right) views of the position evolution of a spacecraft with flybys of Callisto and Io (blue) and with flybys of Callisto, Io, and then another flyby of Io on its second revolution (green). . . . . . . . . .

4.13 Energy versus time (left) and perijove radius versus time (right) of a spacecraft with flybys of Callisto and Io (blue) and with flybys of Callisto, Io, and another flyby of Io on its second revolution (green). . . . . . . . . . . . . .

4.14 Normal (left) and magnified (right) views of the position evolution of a spacecraft with flybys of Callisto and Io (blue) and with flybys of Callisto, Io, and subsequent flybys of Io on each revolution (green) while keeping a constant perijove radius. . .

4.15 Normal (left) and magnified (right) views of energy versus time of a spacecraft with flybys of Callisto and Io (blue) and with flybys of Callisto, Io, and subsequent flybys of Io on each revolution (green) while keeping a constant perijove radius. . . . .

4.16 Perijove radius versus time of a spacecraft with flybys of Callisto and Io (blue) and with flybys of Callisto, Io, and subsequent flybys of Io on each revolution (green) while keeping a constant perijove radius. . . . . . . . . . . . . . .

5.1 Position evolution (left) and eccentricity (right) versus time with an initial apojove radius at Io. The tether is thrusting in green and off in blue. $K_{1}=0.6$ and $K_{2}=0.9 \ldots$. . . .

5.2 Position evolution (left) and eccentricity (right) versus time with an initial apojove radius at Europa. The tether is thrusting in green and off in blue. $K_{1}=1.0$ and $K_{2}=0.7 \ldots$. . . . .

5.3 Position evolution (left) and eccentricity (right) versus time with an initial apojove radius at Ganymede. The tether is thrusting in green and off in blue $K_{1}=0.6$ and $K_{2}=0.9$. .

5.4 Position evolution (left) and eccentricity (right) versus time with an initial apojove radius at Callisto. The tether is thrusting in green and off in blue. $K_{1}=0.7$ and $K_{2}=0.7$. . . .

5.5 Various eccentric orbits in the rotating Sun-Jupiter coordinate frame. . . . . . . . . . . . . . . . 34

5.6 Change in perijove radius versus sun-line angle. . . . . . . . . 35

5.7 Change in perijove radius versus sun-line angle for various sized orbits. . . . . . . . . . . . . . 
5.8 Change in perijove radius versus additional times of flight from a Hohmann transfer for various sized orbits. . . . . . . . . 37

5.9 Lambert-targeted transfer orbits from Earth. . . . . . . . . . 37

5.10 Ballistic trajectory using 28 flybys to raise perijove radius $\left(t_{f}\right.$ $=11$ months $) \ldots \ldots \ldots \ldots \ldots \ldots \ldots$ 


\section{Chapter 1}

\section{Introduction}

Over the past decades, interplanetary missions have shifted from high-thrust to more complex low-thrust, high- $\mathrm{I}_{S P}$ trajectories with multiple flybys. These missions have lower on-board propellant mass requirements in exchange for larger times of flight. There clearly exists a need for future propulsion systems which combine high-thrust capabilities on a direct transfer from Earth, while still remaining low or completely free of propellant requirements. One such proposed propulsion system is the electrodynamic tether, which is capable of generating potentially large amounts of thrust from a planet's magnetosphere.

There exist several planetary bodies in our solar system which possess a magnetic field capable for use by an electrodynamic tether, those being Earth, Jupiter, Saturn, Uranus and Neptune. However, some of these destinations are more attractive than others, with Jupiter being the best choice. It has the strongest planetary magnetic field, is the most accessible (excluding Earth), and it and its moons are ranked highly as targets of interest with regards to planetary science. There are many challenging issues surrounding such a mission, including prohibitive propulsion needs, long trip durations, limited power generation and harsh radiation environments. Although the Galileo and Cassini missions were both successful, their traditional propulsion systems and 
radioisotope thermal generators (RTGs) limited the science return in terms of both payload mass available, due to the large propellant required, and also by limiting the lifetime of the missions [1]. Other proposed missions had sought to use RTGs, nuclear systems or solar arrays for power, though they were considered cost-prohibitive and cancelled [2]. These missions considered both direct and gravity-assisted tours to investigate the trade-off between time of flight and propellant required.

Electrodynamic propulsion systems have the capability to solve or diminish many of the previously mentioned issues. It will be shown that they have the capability to capture into the Jovian system via a direct Hohmann transfer from Earth without the need to add several years onto the mission for gravity assists. Electrodynamic tethers can also be used for both low-thrust propulsion and power generation, which either fulfill the role of the primary or another redundant power generation system once capture in the Jovian system is achieved. Lastly, the system is almost effectively propellant-less, so the amount of onboard fuel is not a driving constraint on the lifetime of the mission.

Electrodynamic tethers have been long considered for Jupiter and other planetary missions [3], and there have been several electrodynamic tether missions conducted in low Earth orbit $[4,5]$. More recently, electrodynamic tethers have been studied in greater depth for capture and tour in the Jovian system $[6,7,8,9]$, though these studies have focused primarily on the capture phase without utilizing flybys of the Jovian moons. Many other Jupiter orbiter studies have been conducted using traditional propulsion systems [10], which 
can give a benchmark for comparison to the electrodynamic tether propulsion mission.

Chapter 2 aims to give a general background on the physics of electrodynamic tethers, and how they can be used to generate thrust in rotating magnetic fields. Chapter 3 describes an overview of the mission architecture for the proposed mission to Jupiter. It also states the assumptions in this work. Chapter 4 through 6 detail the mission trade studies for the capture, apojove lowering, and perijove raising phases of the mission. Lastly, Chapter 7 summarizes the conclusions of this study and gives suggestions for future work. 


\section{Chapter 2}

\section{Physics of Electrodynamic Tethers}

An electrodynamic tether is essentially a long, thin, conducting wire which produces thrust via the Lorentz force. This force is non-conservative and is a function of tether position, velocity and attitude. The induced electric field of a conducting wire moving through a magnetic field is given by:

$$
\bar{E}_{m}=\bar{v}_{r e l} \times \bar{B}
$$

where $\bar{B}$ is the vector of the magnetic field. For the purpose of this analysis, a dipole model is assumed for Jupiter's magnetic field. The magnetic field strength is assumed to follow an inverse cube law. Referenced above, $\bar{v}_{r e l}$ is the velocity of the spacecraft relative to the rotating plasmasphere given by:

$$
\bar{v}_{r e l}=\bar{v}_{S C}-\bar{v}_{p l}
$$

where $\bar{v}_{S C}$ is the spacecraft's velocity vector and $\bar{v}_{p l}$ is the velocity vector of the planet's plasmasphere. The resultant Lorentz force on the conducting wire or tether is:

$$
\bar{F}_{L}=l \bar{I}_{a v g} \times \bar{B}
$$

where $l$ is the length of the tether and $\bar{I}_{\text {avg }}$ is the average current in the wire. This relation is shown in Figure 2.1. 


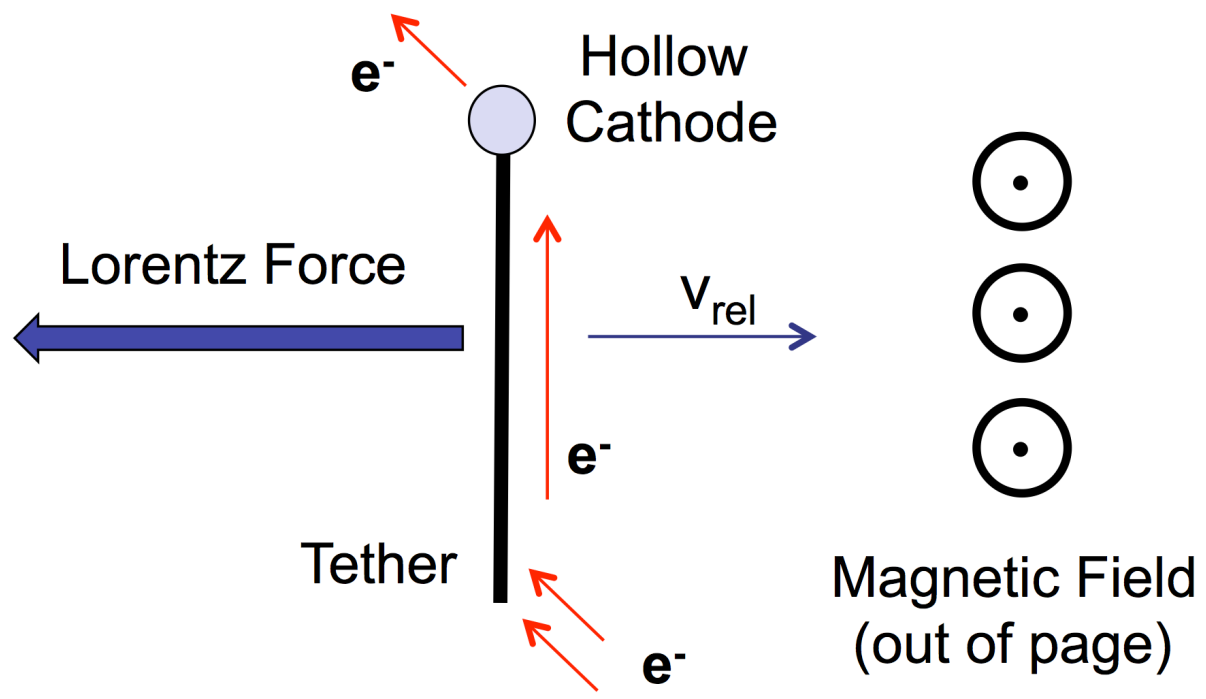

Figure 2.1: Lorentz force generation diagram of conducting wire passing through a planet's magnetic field.

Using a bare tether with width $w$ and choosing the zero-bias point for maximum current, the average current is given as [11]:

$$
\bar{I}_{a v g}=\frac{3}{5} I_{0} \hat{u}
$$

where $\hat{u}$ is the unit vector along the tether and $I_{0}$ is:

$$
I_{0}=\frac{4}{3}\left(\frac{w}{\pi}\right) q_{e} N_{e} l^{\frac{3}{2}} \sqrt{2\left(\bar{E}_{m} \cdot \hat{u}\right)\left(q_{e} / m_{e}\right)}
$$

where $q_{e}$ is the charge of an electron, $m_{e}$ is the mass of an electron and $N_{e}$ is the local plasma electron density, assumed to have a constant value of $3 \times 10^{9}$ $\mathrm{m}^{-3}$. The analysis conducted in the following sections uses a planar two-body gravity model with added Lorentz forces. The tether unit vector, $\hat{u}$ is assumed to point in the direction of maximum generated thrust. 
Shown in Figure 2.2 is the Lorentz force generated by the tether in the Jovian system versus the semi-major axis of the spacecraft's circular orbit plotted on a logarithmic scale. As can be seen, large forces up to hundreds of Newtons can be generated by the tether.

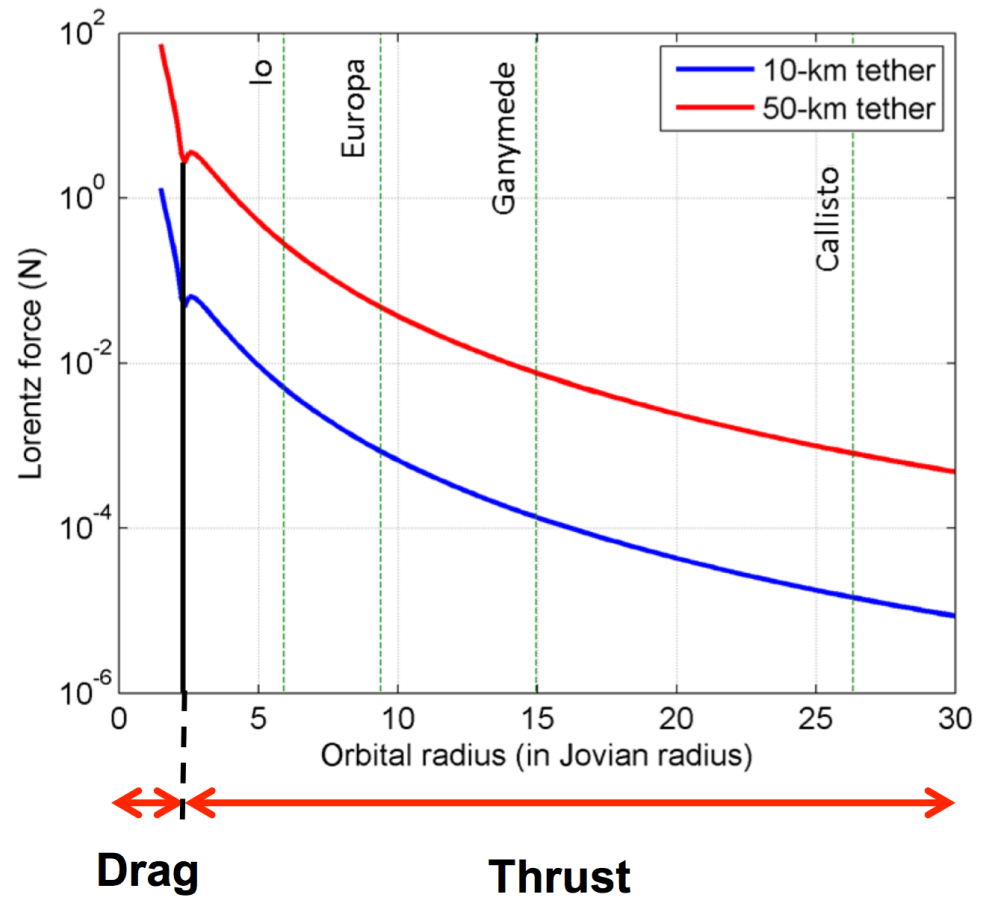

Figure 2.2: Lorentz force generated versus semi-major axis of a circular orbit about Jupiter.

This force then sharply drops off as the relative velocity between the rotating magnetosphere and the spacecraft goes to zero, generating no force. This is referred to as the stationary radius. It should be noted that current can only travel in one direction by nature of the tether design. Thus, when oriented toward the radial direction, a drag force is generated under the stationary 
radius. Past this radius, a thrusting force is generated if the tether is oriented along the anti-radial direction. Both of these forces will be utilized and shown to be useful in the following chapters. 


\section{Chapter 3}

\section{Mission Architecture}

The complexity that arises in designing a Jupiter orbiting and moon tour mission and the corresponding spacecraft should be understated. However, the majority of these aspects will not be covered in this report, but have been touched on in similar studies $[1,12]$. An overview of the mission phases and spacecraft will be briefly discussed here.

\subsection{Mission Phases}

The proposed mission can be organized into four phases, as shown in Figure 3.1. The first phase is the Jupiter orbit insertion maneuver, or capture phase. Capture is clearly the most critical phase, as there will generally be only one opportunity for success. Capture trajectories using both with and without flybys of the Galilean moons will be considered. The next phase, apojove pump-down, will be discussed again considering with and without flybys. The time scale for this phase could be on the order of a year. This could potentially be beneficial, as the long periods of time near apojove and away from the radiation environment could result in an off-loading of some of the accumulated radiation. A detailed study of the radiation dosage will not be presented here. 

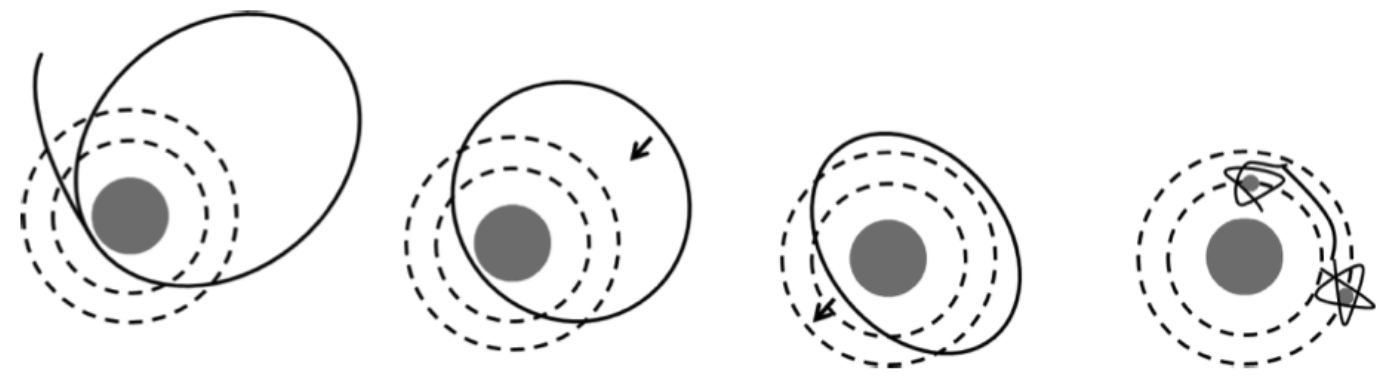

Figure 3.1: Phases of MAGNETOUR mission concept [1] from left to right: capture; apojove pump-down; perijove pump-up; and loosely captured moon tour.

The next phase is perijove pump-up, so as to enable weak capture by one of Jupiter's moons. This phase also has the added benefit of escaping the radiation environment and atmospheric heating effects very close to Jupiter. This and the previous phase of apojove lowering can be thought of collectively as reducing the orbit's eccentricity. Tether thrusting, secondary propulsion systems, and ballistic tours are considered to accomplish this. The use of the third body perturbation from the Sun is also considered.

The goal of the trajectory design phases is to conclude at or near a Hohmann-like orbit between two of the Galilean moons. The spacecraft may then be loosely captured, and the low-energy moon tour may begin. This final moon tour phase will also not be covered here, and is the subject of other works $[13,14,1]$. 


\subsection{Assumptions}

The following analysis makes use of several simplifying assumptions. The dynamics will consider only two-body gravity model and an incoming hyperbolic trajectory given by a Hohmann transfer from Earth. Jupiter's magnetic field, which is tilted at 11 degrees, will be simplified to a perfect dipole centered at Jupiter. As stated earlier, the tether can generate both drag and thrusting forces depending on its orientation. It will be assumed that the tether can optimally oriented at all times. Since this optimal direction is either in the positive or negative radial direction, the gravity gradient may be used to accomplish this. However, the switch from the positive to negative radial direction is not a trivial one. Lastly, a 1,000 kg spacecraft model is assumed, an mockup of which is shown in Figure 3.2.

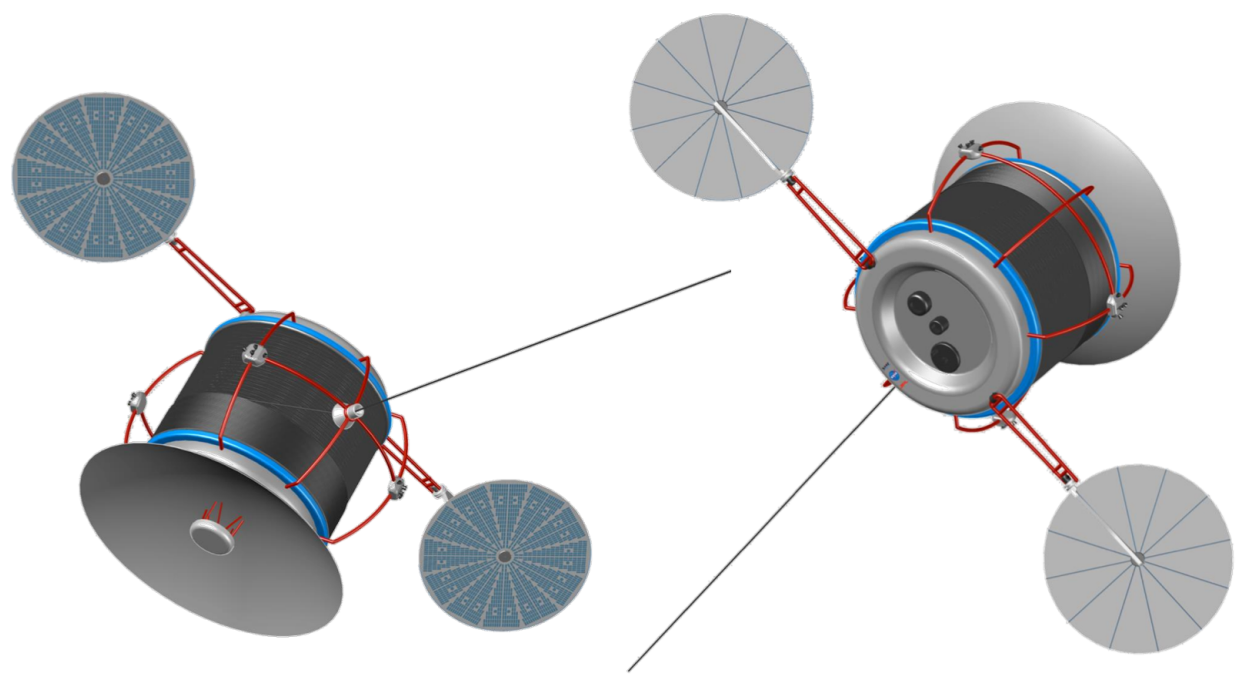

Figure 3.2: Mock-up spacecraft model from the MAGNETOUR mission concept [1], which shows the tether system configuration. 
This spacecraft model assumes an approximately $600 \mathrm{~kg}$ bus and a 200 $\mathrm{kg}$ payload. This leaves approximately $200 \mathrm{~kg}$ for the the tether system. The tether itself is assumed to be a $5 \mathrm{~mm}$ thick tape made of aluminum. 


\section{Chapter 4}

\section{Capture and Apojove Pump-Down}

After Earth departure, the first and most critical phase of a mission using electrodynamic tether propulsion is the magnetobreaking phase to enable capture. The capture problem in the Jovian system is a non-trivial one, as the large hyperbolic velocities would traditionally require a prohibitive amount of onboard fuel or many flybys, resulting in a much longer time of flight. By using an electrodynamic tether propulsion system, this large in-bound velocity can be used to apply a proportional force for the benefit of capture. The Lorentz force is applied in the anti-velocity direction for spacecraft velocities that are greater than the velocity of the plasmasphere, resulting in large $\Delta \mathrm{V}$ changes at perijove. This would potentially make retrograde capture orbits attractive, though this is not necessarily true for touring.

The insertion phase is especially sensitive to the in-bound hyperbola and corresponding perijove radius. The magnetic field strength is a inverse cubic function, and therefore the smaller the perijove radius, the greater the generated Lorentz force. Added to this is the fact that the in-bound velocity of the spacecraft is also higher for a lower perijove. To demonstrate this sensitivity, the capture trajectories for a $50 \mathrm{~km}$ tether spacecraft with varying perijove radii are shown in Figure 4.1. 


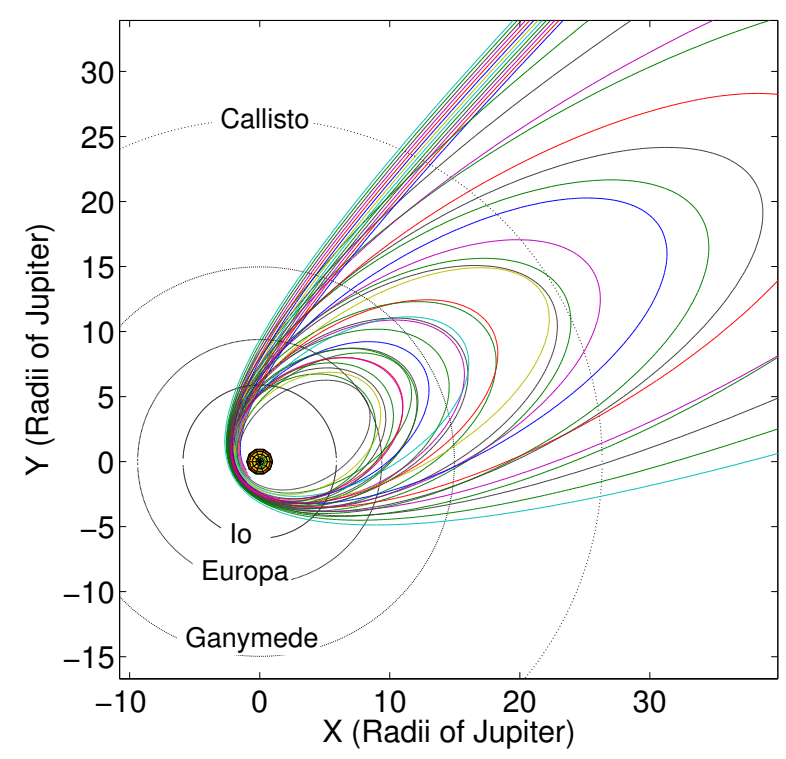

Figure 4.1: Capture orbit evolutions with a $50 \mathrm{~km}$ tether for varying perijove radii.

Therefore, it is obvious that the spacecraft must be captured at the lowest perijove possible. However, the closer proximity to Jupiter brings problems related to heating due to atmospheric drag. A compromise must be made with regards to the radiation dosage and capture performance. It is therefore necessary to find the shortest length tether capable of Jovian capture at a reasonably high perijove radius. It is assumed that it is capable to actively control the tether's orientation such that it remains pointed in the maximum thrust direction. This allows for the maximum amount of thrust, as opposed to using a bang-bang control law [15], resulting in only half the available thrust. The following sections will consider capture trade studies on these trajectories with and without the use of flybys of the Galilean moons. 


\subsection{Capture Without Flybys}

As shown earlier, the capture trajectories are most sensitive to the targeted minimum perijove radius. The Lorentz force generated at this close approach is also proportional to the tether length, as shown in Eq. 2.3. Together, these two quantities become the major design levers in this analysis on capture trajectories.

\subsubsection{Design Space}

The design space was first mapped by varying the inward-bound hyperbolic path and tether length of a 1,000 kg spacecraft, which enables a specific resulting perijove radius to then be targeted. The $\mathrm{V}_{\infty}$ used was $5.64 \mathrm{~km} / \mathrm{s}$, or the equivalent Hohmann transfer from Earth. The total $\Delta \mathrm{V}$ due to the electrodynamic forces during the first close approach was plotted versus the resulting perijove radii for several tether lengths in Figure 4.2. Only solutions that were captured and stayed within Jupiters sphere of influence (SOI) are shown. The minimum perijove radius was chosen at $1.5 R_{J}$.

It is shown that $20 \mathrm{~km}$ tether spacecraft can be just barely captured with a perijove radius of $1.5 \mathrm{R}_{J}$, though the resulting orbit extends far past Jupiters SOI. This length and minimum perijove radius were chosen as benchmark values, along with a $50 \mathrm{~km}$ tether spacecraft, which can reasonably be captured with a perijove radius of $2.0 \mathrm{R}_{J}$. Figure 4.2 also shows the maximum electrodynamic force applied during the close approach versus perijove radius.

After the spacecraft is captured into orbit about Jupiter, the apojove is 

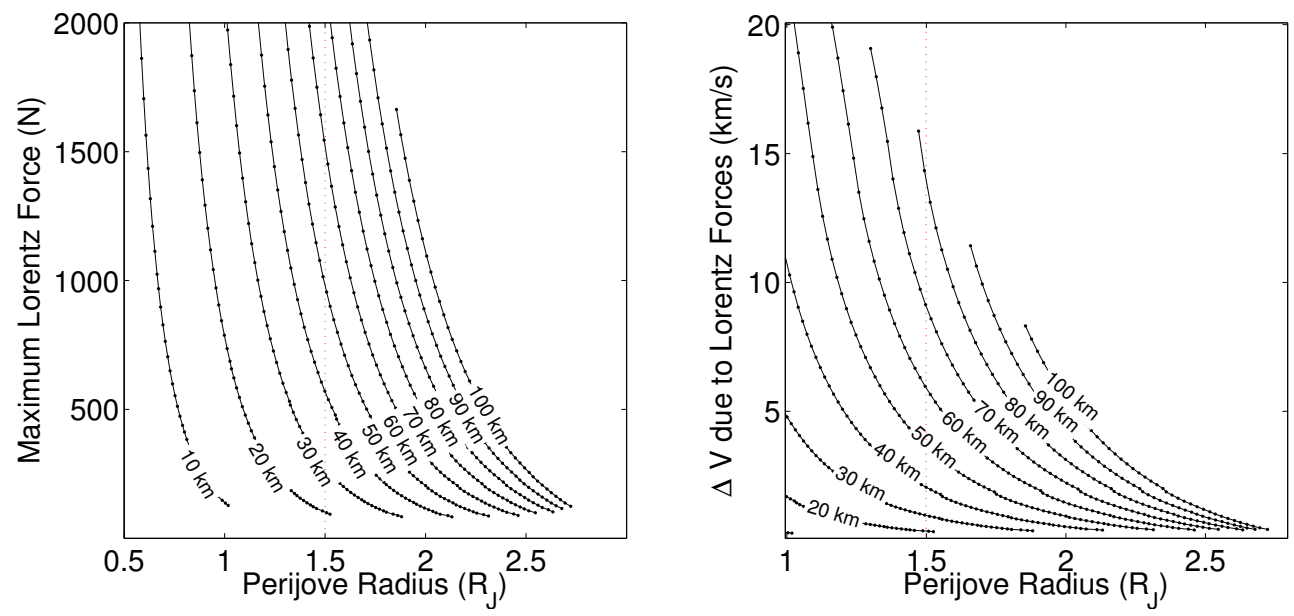

Figure 4.2: The maximum Lorentz force generated during insertion (left) and the corresponding total $\Delta \mathrm{V}$ change (right) for varying perijove radii and tether lengths.

lowered during each revolution by turning on the tether during each perijove pass, resulting in reductions in energy. A desired apojove radius at Callisto $\left(26.34 \mathrm{R}_{J}\right)$ was arbitrarily chosen for comparison purposes. The total time from capture to an orbit with this apojove radius is plotted in Figure 4.3 versus perijove radius for varying length tethers. The desired $20 \mathrm{~km}$ tether spacecraft cannot be captured with a perijove radius of $1.5 \mathrm{R}_{J}$ in under a year. This is remedied in the following section by including flybys of the Jovian moons.

In addition to radiation effects and heating due to atmospheric drag, temperature tolerances in the tether material will also drive the design space. The temperature function is given as:

$$
\left(\frac{2 \epsilon}{h_{t}}\right) \sigma_{B} T^{4}\left(\frac{2 \pi r_{p}}{v_{r e l}}\right)=\left(\frac{M_{S C}}{m_{t}}\right) \rho_{A l} V_{\infty}^{2}
$$




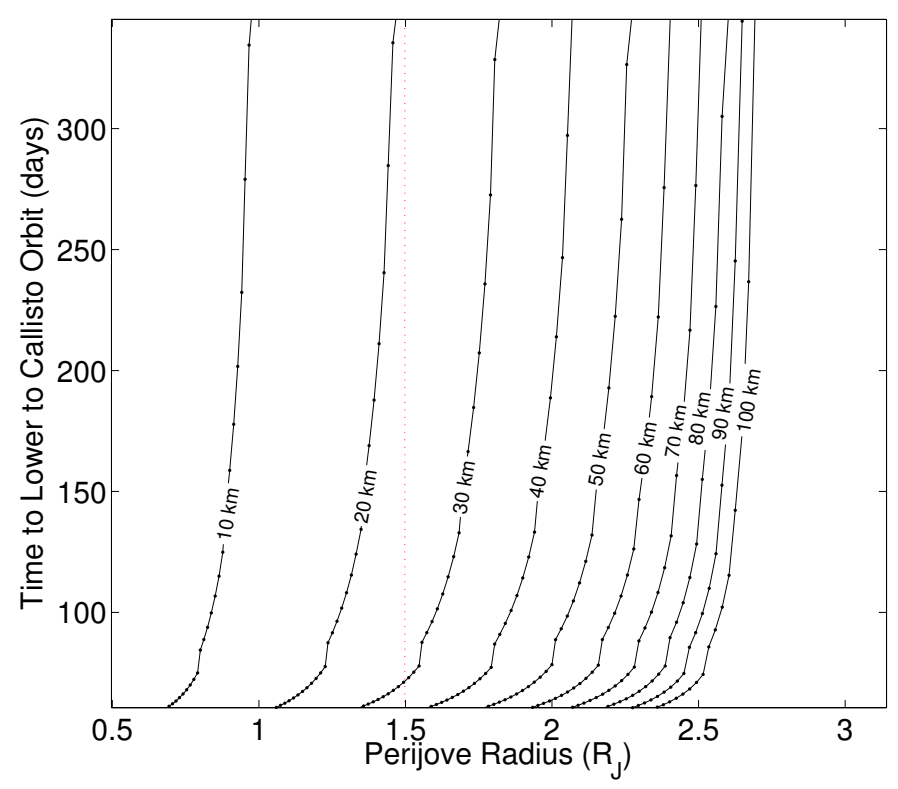

Figure 4.3: The total time needed to capture and lower apojove radius to Callisto for varying perijove radii and tether lengths.

where $\epsilon$ is the emissivity of the tether, $h_{t}$ is the thickness of the tether, $\sigma_{B}$ is the Stefan-Boltzmann constant, $T$ is the temperature in Kelvin, $r_{p}$ is the perijove radius, $M_{S C} / m_{t}$ is the ratio of total mass to tether mass, and $\rho_{A l}$ is the density of the tether material (aluminum) [8]. The maximum temperature due to the impact of electrons collected on the tether is plotted in Figure 4.4 versus perijove radius for varying tether length. Assuming the minimum perijove radius stays above $2.0 \mathrm{R}_{J}$, the maximum temperature of tether stays below a reasonable value of approximately $260 \mathrm{~K}$. Note that this temperature function does not include heating from ohmic dissipation effects. 


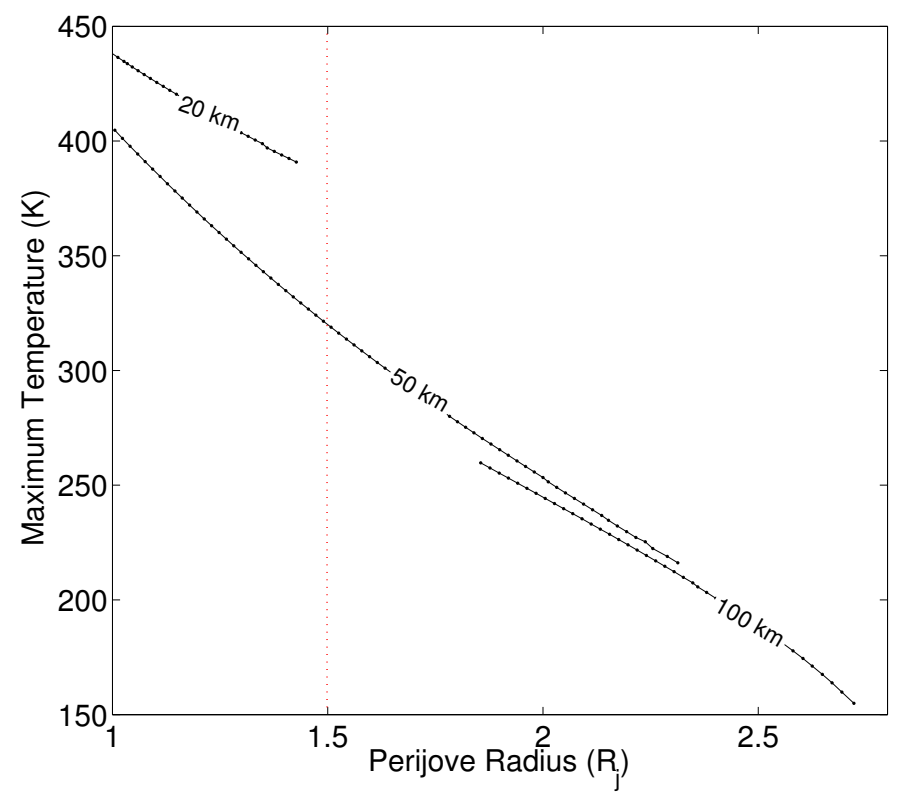

Figure 4.4: The maximum temperature on the tether during insertion for varying perijove radii and tether lengths.

\subsubsection{Baseline Trajectories}

Characteristic trajectories were then simulated using the benchmark values from this analysis. The trajectory of a $50 \mathrm{~km}$ tether with a $2.0 \mathrm{R}_{J}$ apojove radius is plotted in Figure 4.5, with the Lorentz force acting in the anti-velocity direction versus time given in Figure 4.6. This spacecraft would indeed become captured in the Jupiter system, with an equivalent $\Delta \mathrm{V}$ burn of $0.83 \mathrm{~km} / \mathrm{s}$ applied at perijove, and would be lowered to Europa in less than 100 days.

Another baseline value was chosen as $20 \mathrm{~km}$ tether with a minimum perijove radius of $1.5 \mathrm{R}_{J}$. The trajectory of this case is plotted in Figure 4.7, with the Lorentz force acting in the anti-velocity direction versus time given in 


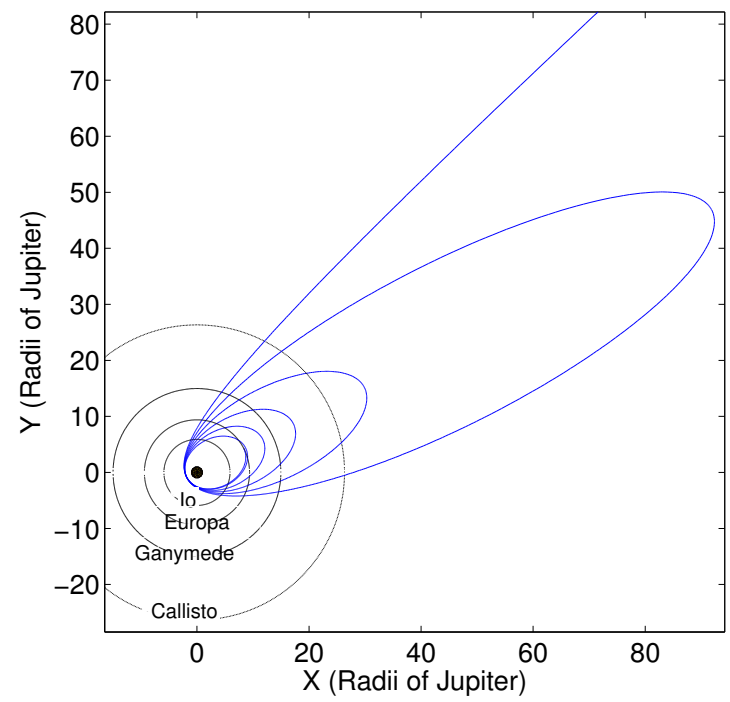

Figure 4.5: Characteristic trajectory for a $50 \mathrm{~km}$ tether spacecraft with a perijove radius of $2.0 \mathrm{R}_{J}$.
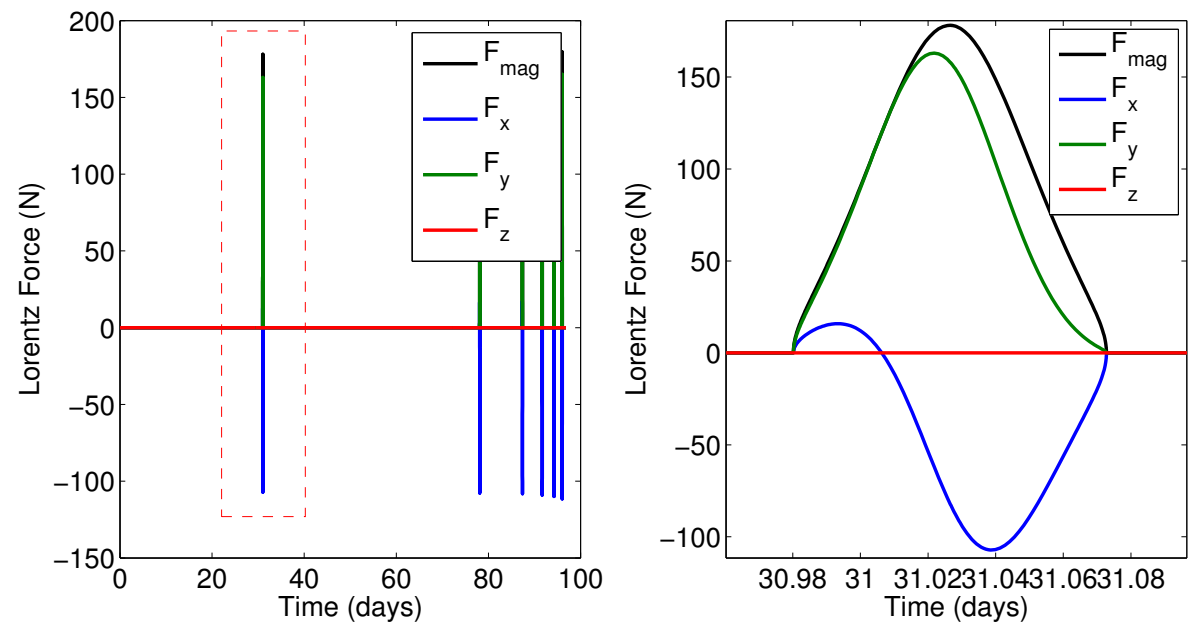

Figure 4.6: Normal (left) and magnified (right) views of the Lorentz force versus time for a $50 \mathrm{~km}$ tether with a periojve radius of $2.0 \mathrm{R}_{J}$. 
Figure 4.8. This spacecraft would also become captured, although its resulting orbit would have an apojove radius outside of Jupiter's sphere of influence. Together, these baseline cases represent the lower bounds on the capture performance achievable with only the use of the tether force.

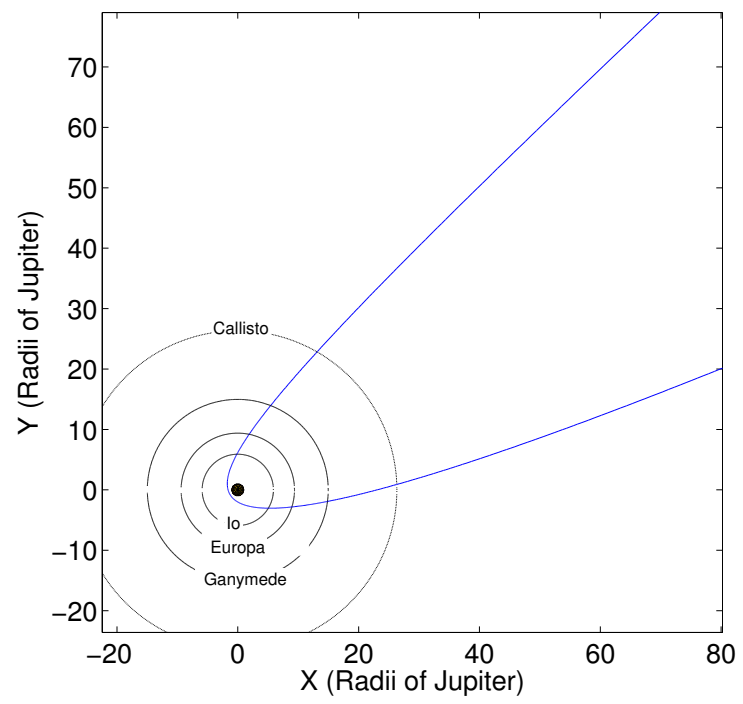

Figure 4.7: Characteristic trajectory for a $20 \mathrm{~km}$ tether spacecraft with a perijove radius of $1.5 \mathrm{R}_{J}$. 

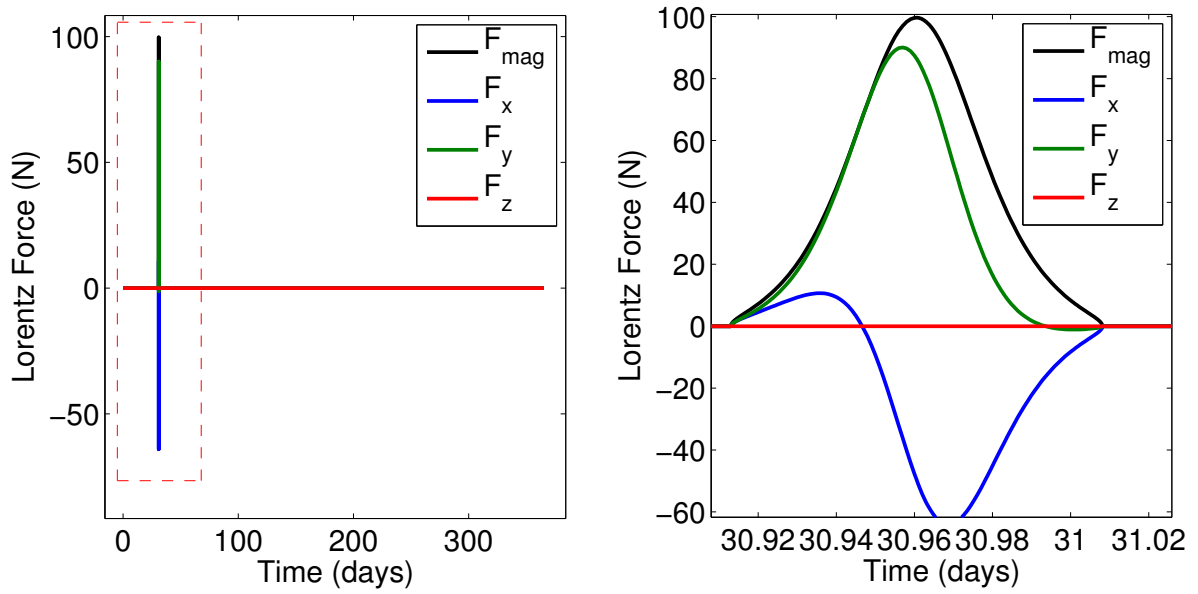

Figure 4.8: Normal (left) and magnified (right) views of the Lorentz force versus time for a $20 \mathrm{~km}$ tether with a periojve radius of $1.5 \mathrm{R}_{J}$.

\subsection{Capture With Flybys}

While capture using only the electrodynamic forces on the spacecraft was shown to be feasible with a tether of suitable length, it is obvious that better solutions that utilize flybys of the Jovian moons exist. Flyby maneuvers were added to the simulation for this analysis using a phase-free assumption for simplicity, which represents the best-case scenario. At each specified intersection of the moons orbit, the $\mathrm{V}_{\infty}$ vector is rotated in the direction that decreases the spacecrafts velocity, as shown in Figure 4.9. The altitude of each flyby is given as $100 \mathrm{~km}$.

\subsubsection{In-Bound Flybys}

With the lower bounding solution being the trajectory that does no flyby maneuvers, the upper bounding case of four consecutive flybys of the Jovian 


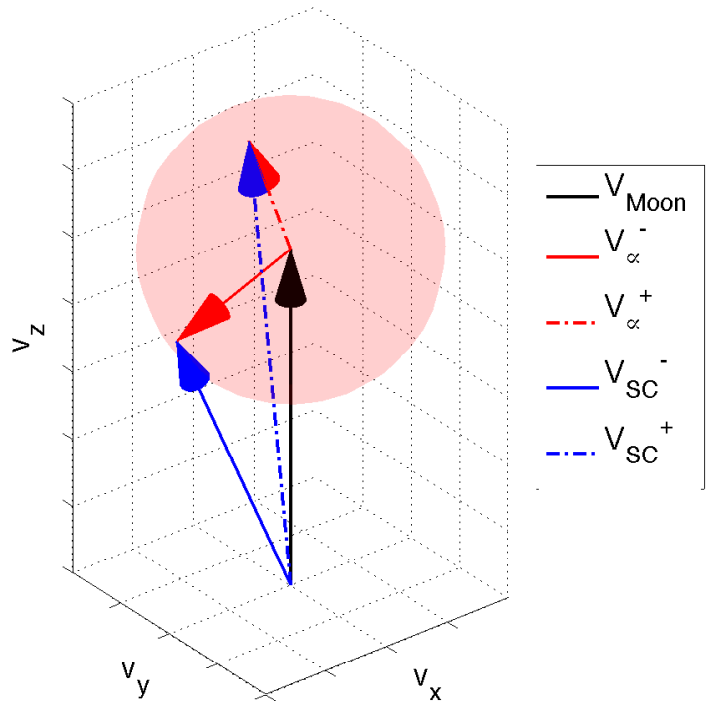

Figure 4.9: Vector diagram showing $\mathrm{V}_{\infty}$ and spacecraft velocity vectors before the flyby and the corresponding vectors after the flyby (not to scale).

moons was found using a $20 \mathrm{~km}$ tether. The perijove radius on the final revolution after lowering the apojove to Europa was targeted as $1.5 \mathrm{R}_{J}$. This trajectory is plotted in Figure 4.10 in comparison with no flyby solution. The energy is plotted versus time in Figure 4.11.

The energy decrease from the four flybys is comparable to energy decrease due to the tether alone in the no flyby case. Note that the energy decrease in the four flyby case is smaller than that in the no flyby case; this is due to the lower spacecraft velocity at perijove. 


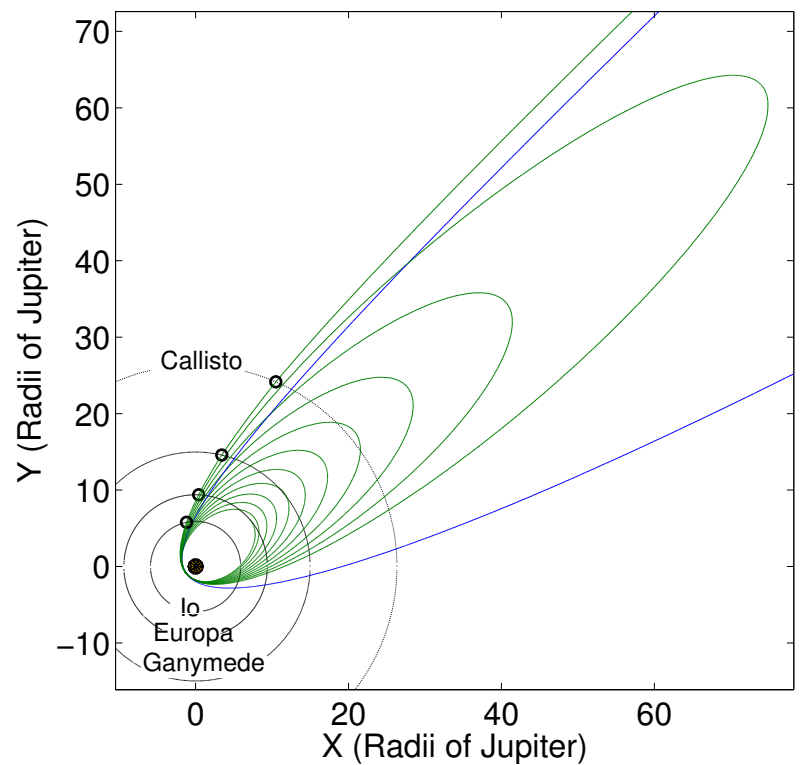

Figure 4.10: The position evolution of a spacecraft with no flybys (blue) and with flybys of Callisto, Ganymede, Europa and Io (green).

\subsubsection{Out-Bound Flybys}

The benefits of flybys on the inward bound leg of the hyperbola are easily seen, however, flybys during the subsequent revolutions have the negative effect of lowering perijove by a much larger amount than simply from the tether thrusting. This is due to the rotation of the velocity vector. If the same final perijove radius is to be targeted, then the perijove on previous revolutions will have to be greater than if there is to be no flyby. This results in smaller electrodynamic force contribution and larger total time to lower apojove radius.

Plotted in Figure 4.12 are two more realistic trajectories: one with flybys of Callisto and Io, and the other with flybys of Callisto, Io and Io again 

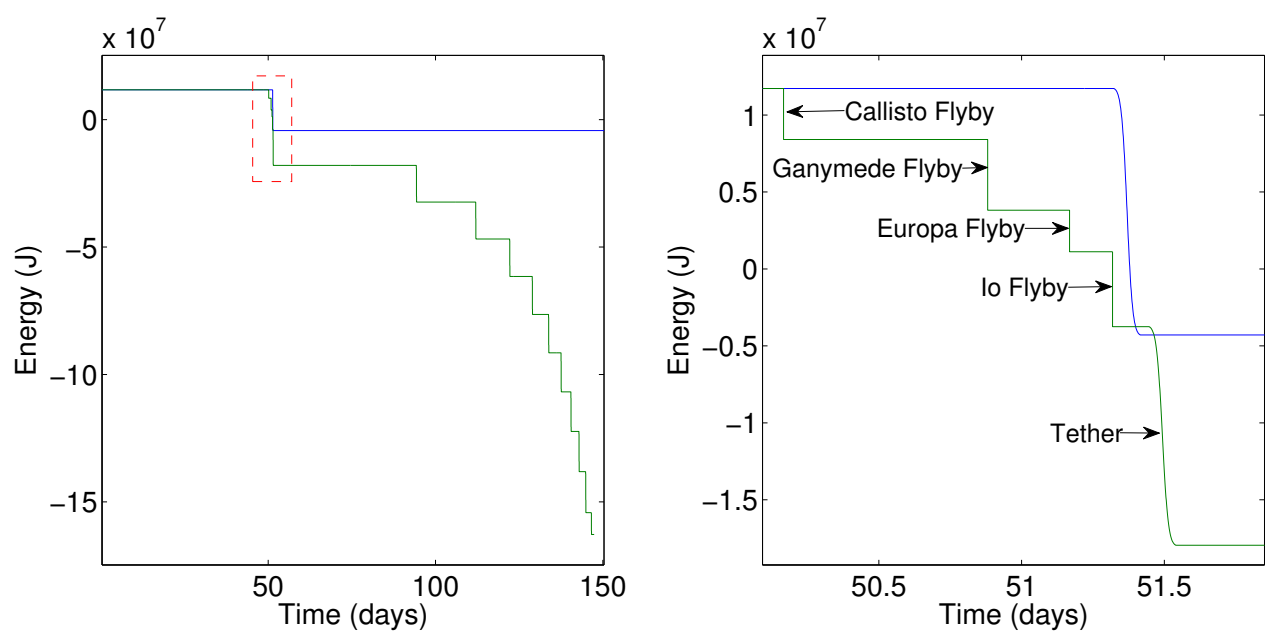

Figure 4.11: Normal (left) and magnified (right) plots of energy versus time of a spacecraft with no flybys (blue) and with flybys of Callisto, Ganymede, Europa and Io (green).

on the spacecrafts second revolution. If the same final perijove radius is to be targeted, it is shown that the first case of only the two flybys is optimal with a larger time-savings shown on the first revolution. This effect is due to the fact that the electrodynamic force is the dominating term in this region.

The energy versus time and perijove radius versus time plots for each trajectory are plotted in Figure 4.13. It was found that by adding the second flyby of Io, the lowering the apojove to the desired value took approximately 50 days longer to achieve.

\subsubsection{Out-Bound Flybys with Constant Perijove}

An alternative strategy capable of taking advantage of flybys on subsequent revolutions would utilize an on-board propulsion system. This propulsion sys- 

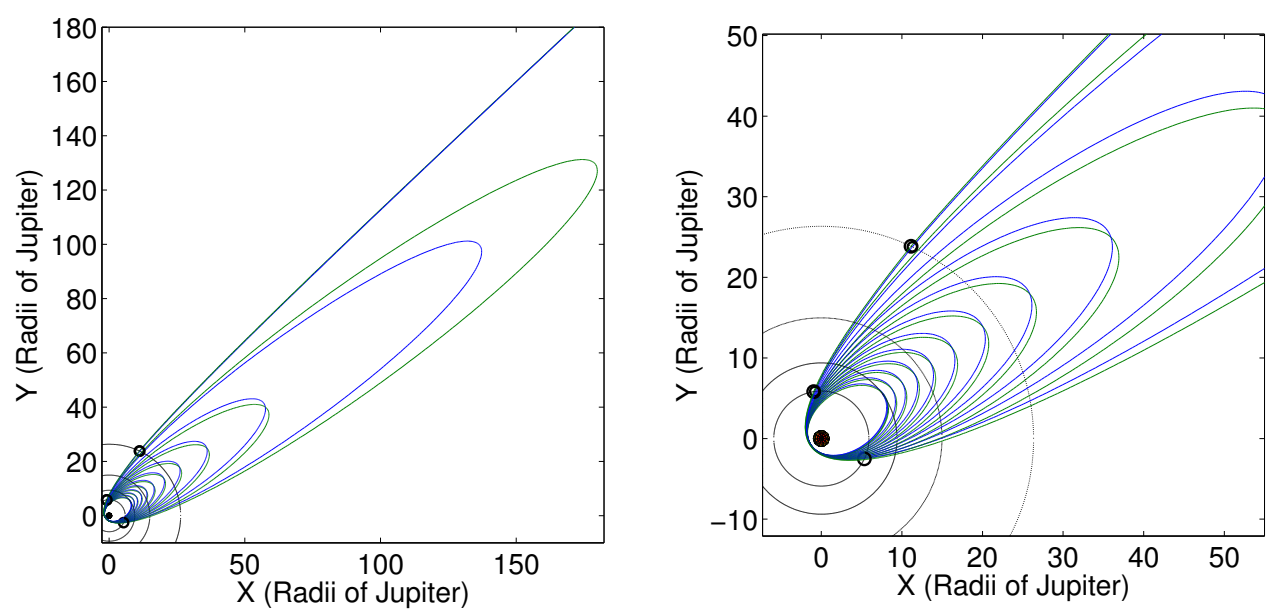

Figure 4.12: Normal (left) and magnified (right) views of the position evolution of a spacecraft with flybys of Callisto and Io (blue) and with flybys of Callisto, Io, and then another flyby of Io on its second revolution (green).

tem, either electric or conventional, would raise perijove radius on each pass through apojove. This method would keep the effective perijove distance constant as long as the subsequent flybys occur on the outward-bound half of the orbit ellipse.

A case study was done comparing the solution with flybys of Callisto and Io with the solution with flybys of Callisto, Io, and subsequent flybys of Io on each revolution. The perijove radius of the second solution is kept constant by applying a $\Delta \mathrm{V}$ burn at apojove. Both solutions assume a $20 \mathrm{~km}$ tether spacecraft and are targeted for a final perijove radius of $1.5 \mathrm{R}_{J}$. Figure 4.14 shows the position evolution of both of these trajectories. 

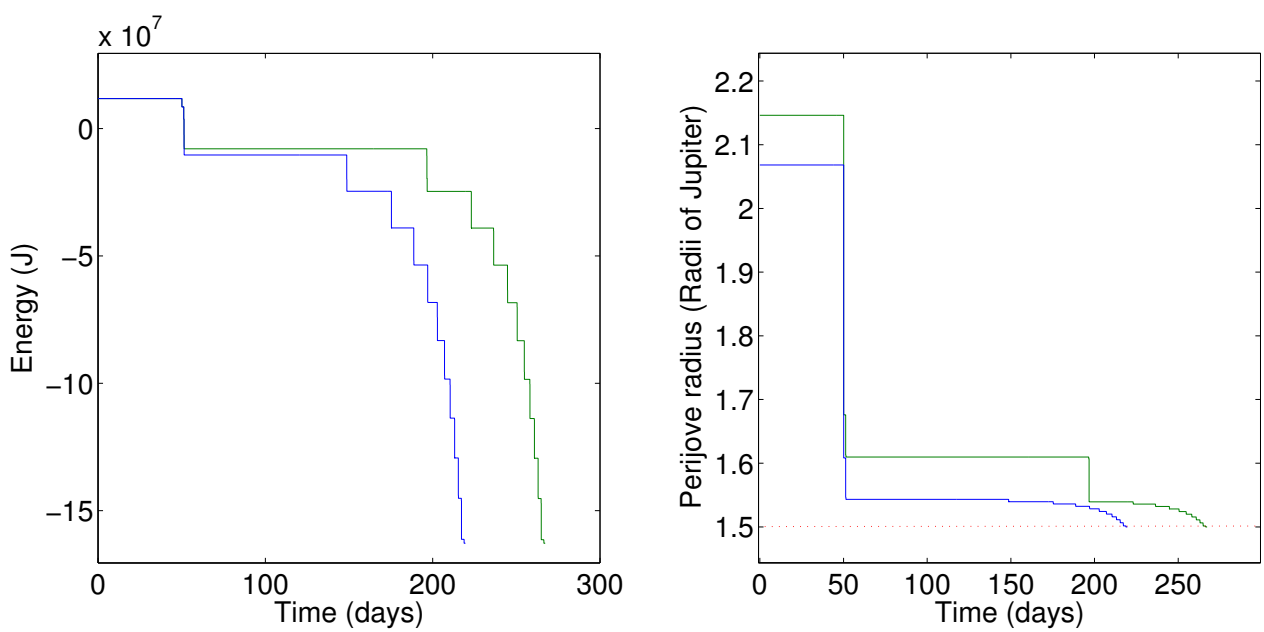

Figure 4.13: Energy versus time (left) and perijove radius versus time (right) of a spacecraft with flybys of Callisto and Io (blue) and with flybys of Callisto, Io, and another flyby of Io on its second revolution (green).
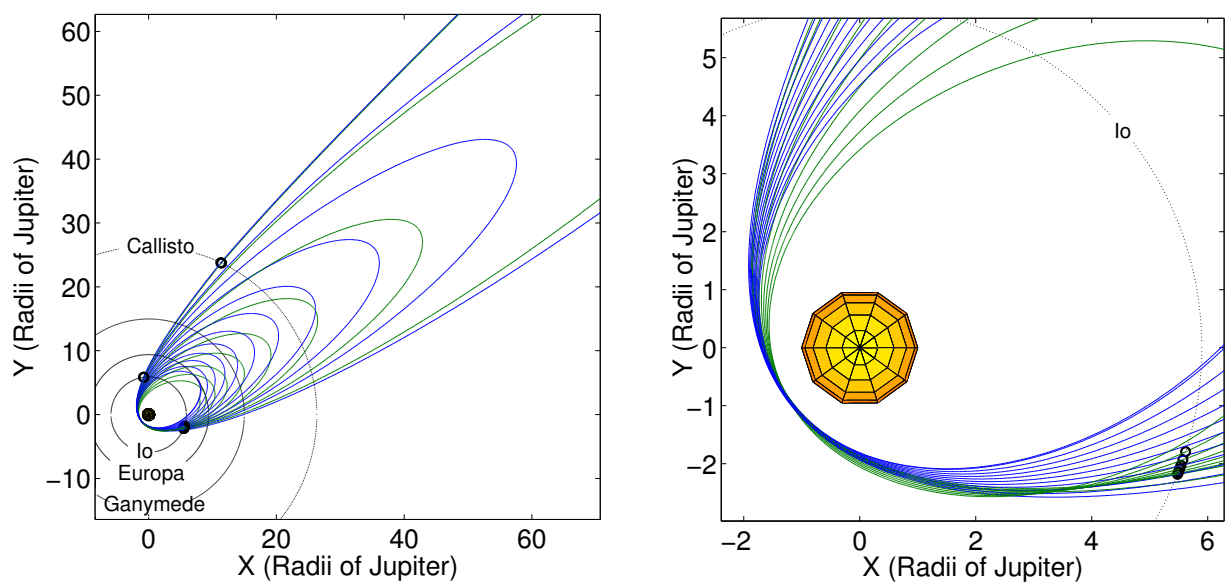

Figure 4.14: Normal (left) and magnified (right) views of the position evolution of a spacecraft with flybys of Callisto and Io (blue) and with flybys of Callisto, Io, and subsequent flybys of Io on each revolution (green) while keeping a constant perijove radius. 

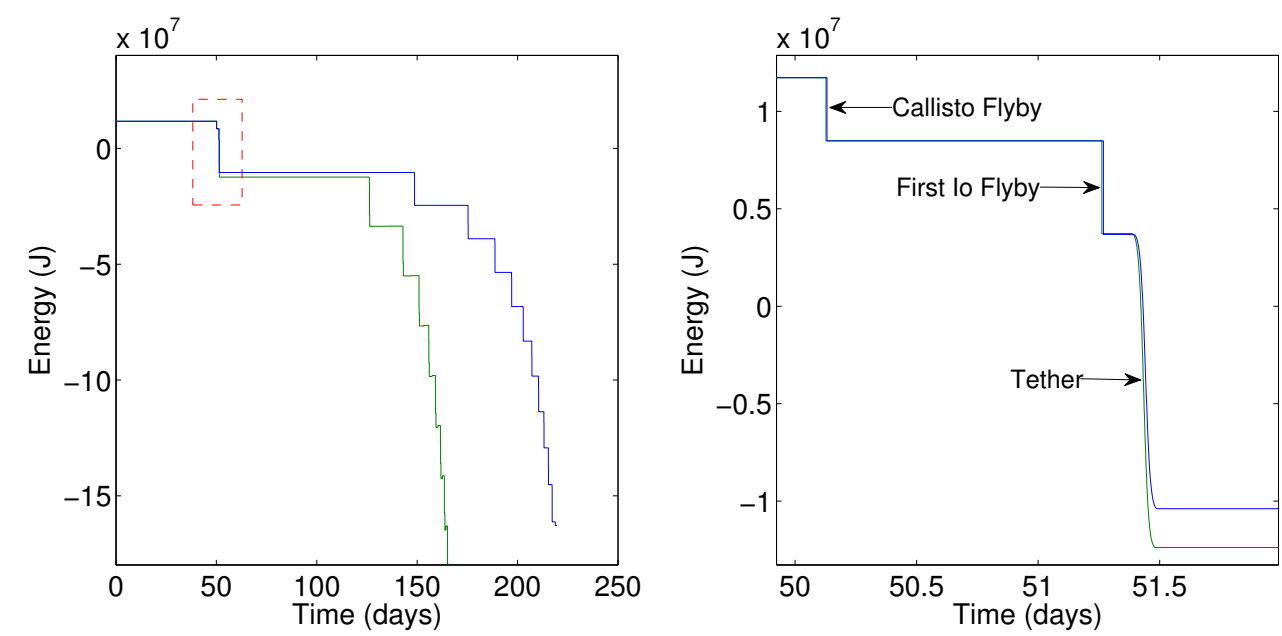

Figure 4.15: Normal (left) and magnified (right) views of energy versus time of a spacecraft with flybys of Callisto and Io (blue) and with flybys of Callisto, Io, and subsequent flybys of Io on each revolution (green) while keeping a constant perijove radius.

The required $\Delta \mathrm{V}$ burns at apojove to keep the constant perijove and the efficiency of the burns for each revolution are shown in Table 4.1. It is shown to be less advantageous to continue to do flyby maneuvers as the energy becomes increasingly negative, as the $\Delta \mathrm{V}$ required doing so grows to unreasonably high values and the normalized efficiency of each burn decreases. Therefore, it recommended that $\Delta \mathrm{V}$ burns be performed only on the first or second revolutions.

The corresponding energy versus time plot for both trajectories is shown in Figure 4.15. The second solution (with Io flybys on each revolution) is capable of lowering its energy in approximately 50 less days than without subsequent flybys. This savings comes at the cost of additional total $\Delta \mathrm{V}$ of 627.27 $\mathrm{m} / \mathrm{s}$. The perijove radius versus time for both trajectories is plotted in Fig- 


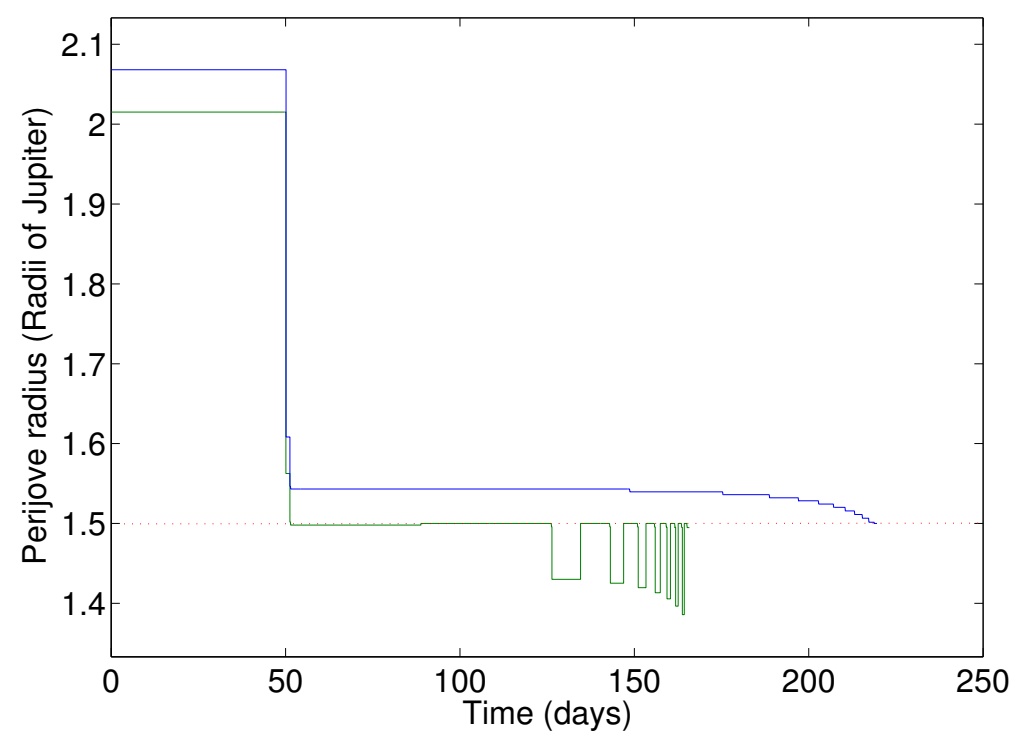

Figure 4.16: Perijove radius versus time of a spacecraft with flybys of Callisto and Io (blue) and with flybys of Callisto, Io, and subsequent flybys of Io on each revolution (green) while keeping a constant perijove radius.

Table 4.1: The required $\Delta \mathrm{V}$ burns applied at apojove to keep a constant perijove of $1.5 \mathrm{R}_{J}$ for each revolution.

\begin{tabular}{|l|c|c|c|c|c|c|c|}
\hline Revolution & 1 & 2 & 3 & 4 & 5 & 6 & 7 \\
\hline $\begin{array}{l}\Delta \mathbf{V} \text { Required } \\
\text { at Apojove } \\
(\mathbf{m} / \mathbf{s})\end{array}$ & 0.33 & 32.13 & 55.75 & 82.70 & 113.75 & 149.95 & 192.66 \\
\hline $\begin{array}{l}\Delta \text { Energy per } \\
\text { Rev. (MJ) }\end{array}$ & 9.0 & 6.9 & 6.9 & 6.9 & 6.8 & 6.8 & 6.6 \\
\hline $\begin{array}{l}\text { Normalized } \\
\text { Efficiency of } \\
\text { Burn, } \boldsymbol{\zeta} \\
(\Delta \mathbf{E} / \Delta \mathbf{V})\end{array}$ & 1.0 & $7.8 \mathrm{e}-3$ & $4.5 \mathrm{e}-3$ & $3.1 \mathrm{e}-3$ & $2.2 \mathrm{e}-3$ & $1.7 \mathrm{e}-3$ & $1.3 \mathrm{e}-3$ \\
\hline
\end{tabular}

ure 4.16. In conclusion, a $20 \mathrm{~km}$ spacecraft is capable of capture in the Jovian system, albeit in a large period orbit. The addition of flybys on the inward- 
bound path gives a free performance increase, although subsequent flybys may not be beneficial. 


\section{Chapter 5}

\section{Perijove Pump-Up}

After the energy of the spacecraft is lowered a sufficient amount, the next step is to raise the spacecraft's perijove such that it is out of the radiation environment near Jupiter and able to become weakly captured by one of its moons. This phase is ideally performed as quickly as possible, so as to limit the accumulated radiation dosage. In this section, raising perijove via the tether force, third body perturbations, and flybys will be considered.

\subsection{Tether Control Law}

The most attractive method to raise perijove is to use only the Lorentz force tether. This does not require any additional propellent, and although it is conceptually simple to imagine (ie. thrust along the velocity vector at apojove), in reality is much more complicated. The typical low-thrust method to raise perijove is not applicable here, as the thrusting direction is constrained and the magnitude is a function of both distance and velocity. An optimal control law was not developed in this study; instead, a heuristic control law was used:

$$
\left(|\hat{r} \cdot \hat{v}|<K_{1}\right) \wedge\left(|\bar{r}|>K_{2} \cdot a_{\text {orbit }}\right) \Longrightarrow \bar{\tau}=\bar{\tau}_{\max }
$$

where $a_{\text {orbit }}$ is the semi-major axis, $\bar{\tau}_{\max }$ is the maximum thrust vector and $K_{1}$ 
and $K_{2}$ are constants to be determined. This heuristic control law ensures that the tether will be thrusting near perijove and that this window for applying thrust grows gradually as the eccentricity of the orbit is lowered.

An ideal initial apojove radius is one at Europa, as the force available decreases with distance and Io does not have a suitable radiation environment. Starting at Io also gives diminishing gains, as thrusting far from apojove also increases apojove radius. Shown in Figure 5.1 is an example of this perijove raising control law starting with an initial apojove radius at Io and with a final time of 1 year. The corresponding plot of eccentricity versus time is also shown.
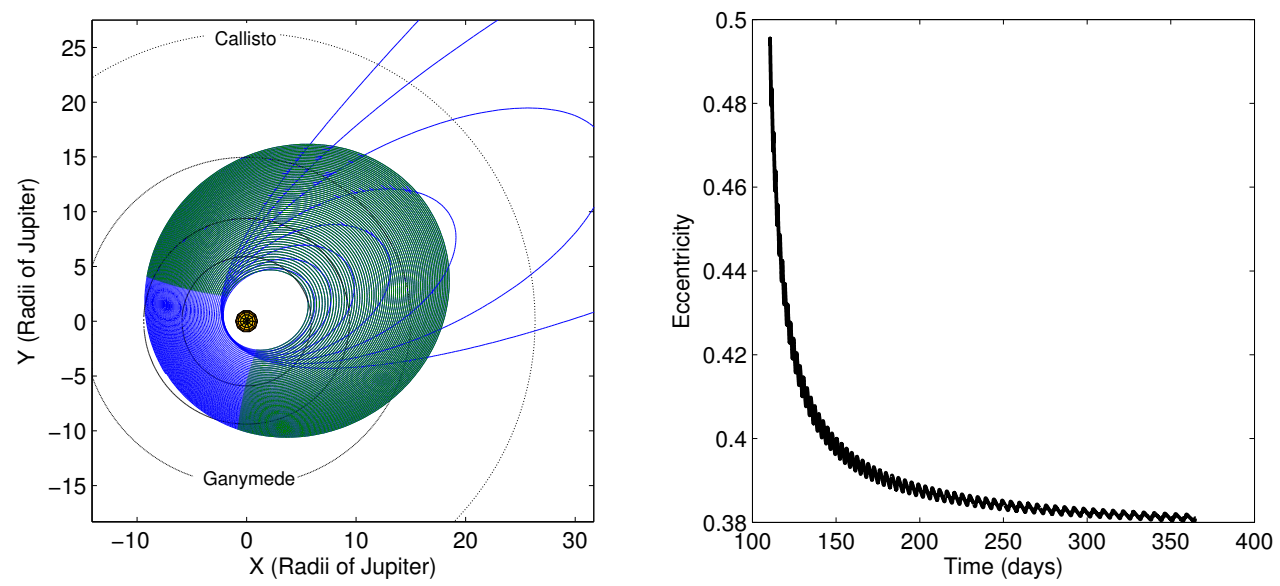

Figure 5.1: Position evolution (left) and eccentricity (right) versus time with an initial apojove radius at Io. The tether is thrusting in green and off in blue. $K_{1}=0.6$ and $K_{2}=0.9$.

The Lorentz force available at Europa with a $50 \mathrm{~km}$ tether spacecraft is on the order of $1 \mathrm{~N}$, and gives much more favorable return on perijove radius 
increase. Figure 5.2 is an example of the perijove raising control law starting with an initial apojove radius at Europa and with a final time of 1 year. The corresponding plot of eccentricity versus time is also shown.
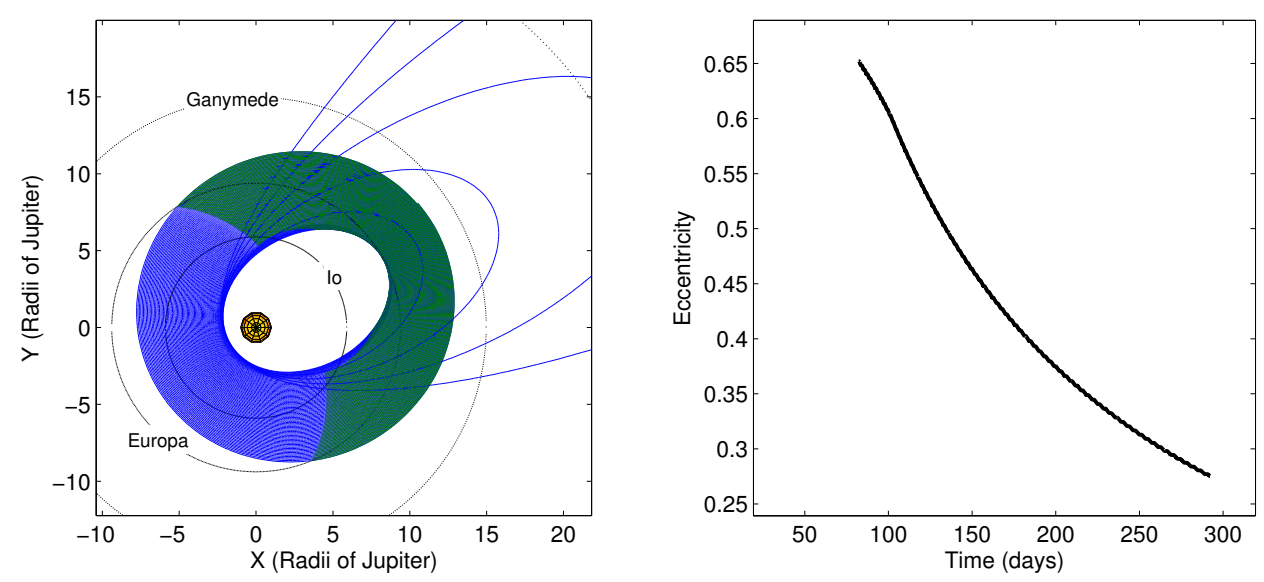

Figure 5.2: Position evolution (left) and eccentricity (right) versus time with an initial apojove radius at Europa. The tether is thrusting in green and off in blue. $K_{1}=1.0$ and $K_{2}=0.7$.

The final two equivalent plots of the perijove raising control law starting with an initial apojove radius at Ganymede and Callisto are shown in Figure 5.3 and Figure 5.4, respectively. The corresponding plots of eccentricity versus time are also shown. The force available at a distance at Callisto is only approximately $10 \mathrm{mN}$. Starting at Callisto is not a viable option because of this weak Lorentz force available and also a performance decrease resulting from longer orbital periods.

A grid search on constants $K_{1}$ and $K_{2}$ was conducted to find the maximum perijove radius achieved after one year of maneuvering. Table 5.1 shows 

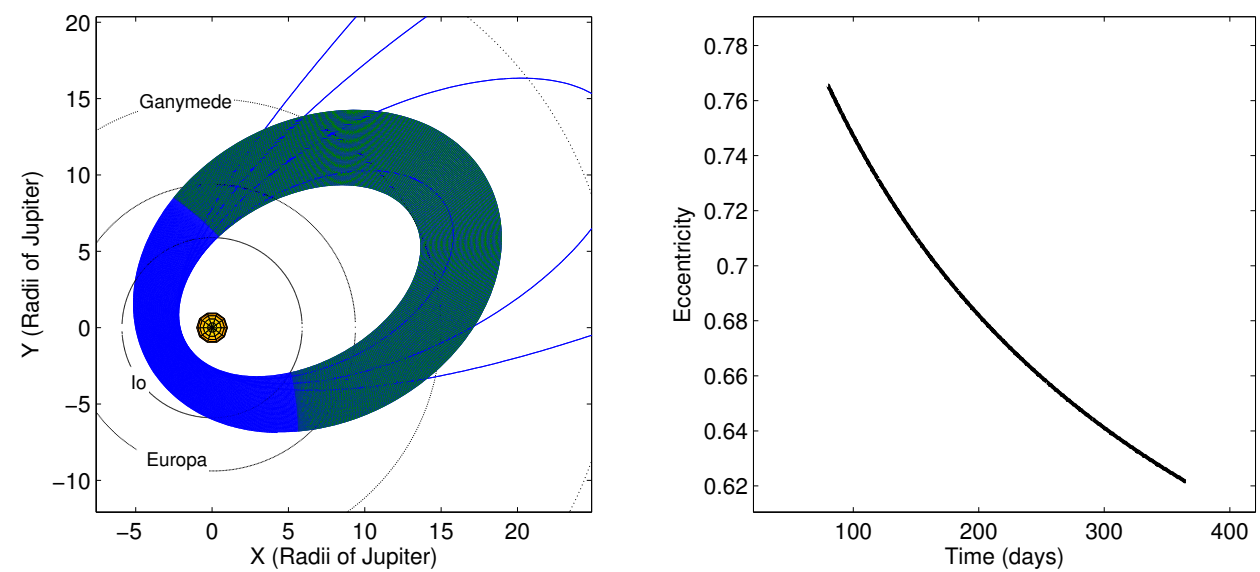

Figure 5.3: Position evolution (left) and eccentricity (right) versus time with an initial apojove radius at Ganymede. The tether is thrusting in green and off in blue $K_{1}=0.6$ and $K_{2}=0.9$.
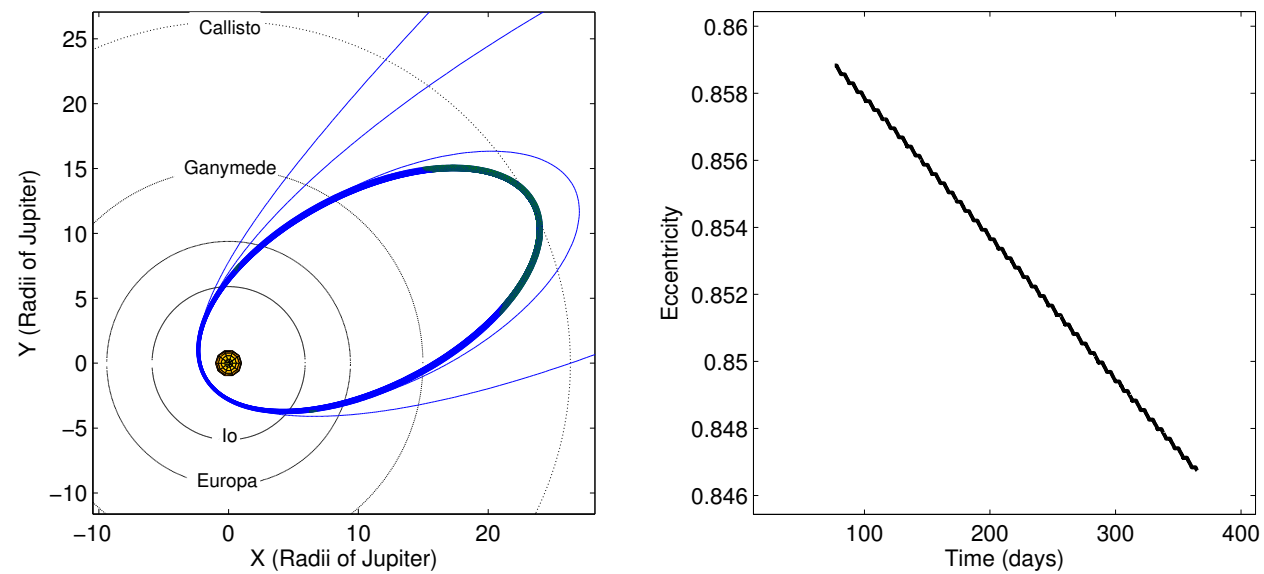

Figure 5.4: Position evolution (left) and eccentricity (right) versus time with an initial apojove radius at Callisto. The tether is thrusting in green and off in blue. $K_{1}=0.7$ and $K_{2}=0.7$. 
the maximum values possible after 1 year using initial apojove radii at each of the Jovian moons. Raising the perijove radius of the orbit using the tether alone may be a possibility depending on the initial orbit and radiation tolerances of the spacecraft. The best case scenario, targeting an apojove radius at Europa, can result in a Ganymede-Europa Hohmann orbit in 1.3 years after reaching Jupiter's sphere of influence.

Table 5.1: Maximum perijove radius achieved after 1 year on $\left[K_{1}, K_{2}\right]$, starting with apojove radius at each Jovian moon.

\begin{tabular}{|l|c|c|c|c|}
\hline $\begin{array}{l}\text { Initial Apojove } \\
\text { Radius }\left(R_{J}\right)\end{array}$ & $\begin{array}{c}26.34 \\
(\text { Callisto) }\end{array}$ & $\begin{array}{c}14.97 \\
(\text { Ganymede) }\end{array}$ & $\begin{array}{c}9.39 \\
\text { (Europa) }\end{array}$ & $\begin{array}{c}5.90 \\
(\text { Io })\end{array}$ \\
\hline $\begin{array}{l}\text { Initial Perijove } \\
\text { Radius }\left(R_{J}\right)\end{array}$ & 2.00 & 2.00 & 2.00 & 2.00 \\
\hline $\begin{array}{l}\text { Final Apojove } \\
\text { Radius }\left(R_{J}\right)\end{array}$ & 26.78 & 20.47 & 13.32 & 19.34 \\
\hline $\begin{array}{l}\text { Final Perijove } \\
\text { Radius }\left(R_{J}\right)\end{array}$ & 3.03 & 4.64 & 8.62 & 8.80 \\
\hline $\begin{array}{l}\text { Location of } \\
\text { Maximum, } \\
{\left[K_{1}, K_{2}\right]}\end{array}$ & {$[0.8,0.5]$} & {$[1.0,0.7]$} & {$[0.6,0.9]$} & {$[0.7,0.7]$} \\
\hline
\end{tabular}

\subsection{Third Body Perturbations}

Another consideration in raising perijove is to leverage the net effects of the solar third-body perturbations. This effect could also potentially be used to hold the perijove constant, as discussed in the previous chapter. This cost-free effect is accomplished by orienting the capture and subsequent revolutions in such a manner that the net force raises perijove [16]. This is shown in Figure 5.5. 


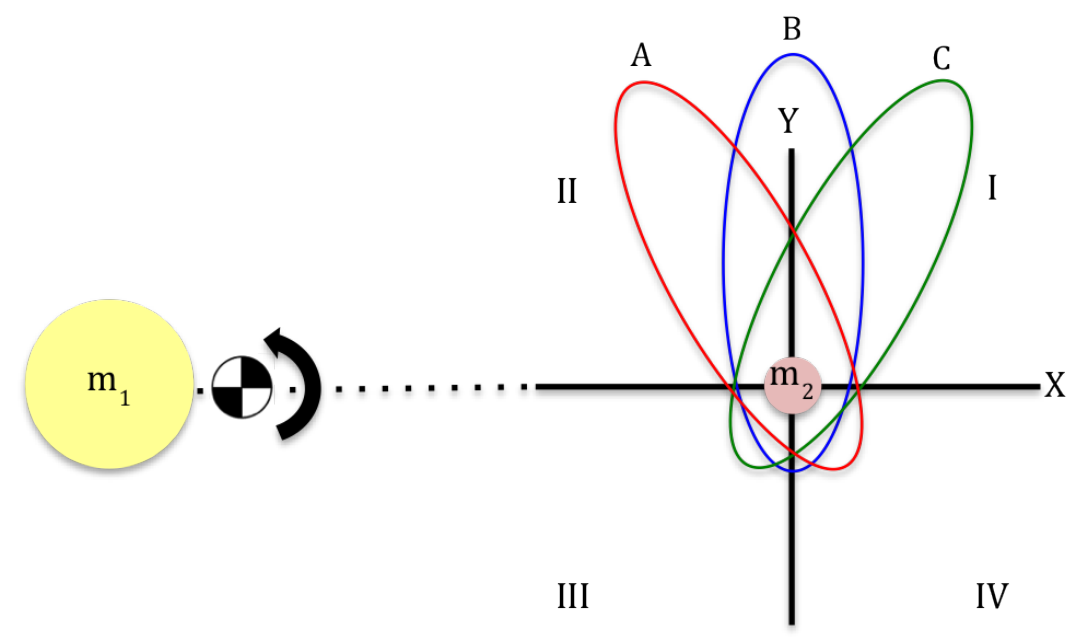

Figure 5.5: Various eccentric orbits in the rotating Sun-Jupiter coordinate frame.

The solar perturbation increases perijove radius if the spacecraft's apojove is located in quadrants II or IV, such as for orbit A (red), while there is a decrease in perijove radius if apojove is located in quadrants I or III, such as for orbit $\mathrm{C}$ (green). There is no net effect on orbit B (blue). This net effect over time is not zero because as the orbit precesses about Jupiter, the apojove radius is being lowered at the same time. Therefore, the tidal effects are greatest for longer period orbits with higher eccentricities. The net change in perijove radius over 100 days versus the sun-line angle (angle counter-clockwise from the negative $\mathrm{X}$-axis in Figure 5.5) for an orbit with apojove at Europa is shown in Figure 5.6.

The sinusoidal effect is clearly seen, with the greatest net effects at 45 degrees in each quadrant. The desired apojove for this application will be 


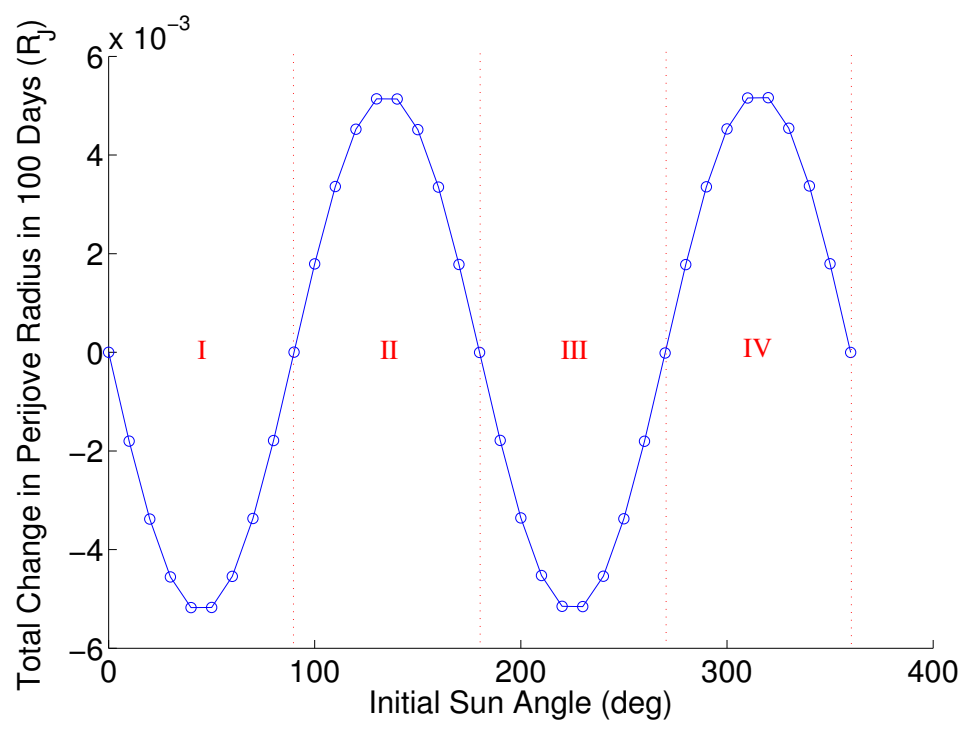

Figure 5.6: Change in perijove radius versus sun-line angle.

found in quadrant IV. Shown in Figure 5.7 are net effects on perijove radius over one year versus a reasonable range of initial sun-line angles for several different sized orbits. This cost-benefit is better analyzed by plotting the net effects versus the extra time of flight required relative to the Hohmann transfer from Earth. Shown in Figure 5.8 is the change in perijove radius in one year versus the additional time required from Hohmann. The actual net effect resulting from a Hohmann transfer is negative, as the resulting ellipse does not perfectly align with the asymptote of the in-bound hyperbola. This angle between the in-bound $\mathrm{V}_{\infty}$ vector and the orbit's apse line varies based on tether length and perijove radius, but is roughly 10 to 15 degrees. Therefore, to counteract this small but unfavorable effect, three extra months of flight time are required reach a neutral effect. Also, an additional 1-2 years of 
flight time are required to receive any sort of useful benefit from the solar 3rd body perturbation. The reason for this is shown Figure 5.9, which gives the discretized Lambert solutions that are required for the desired sun-line angles.

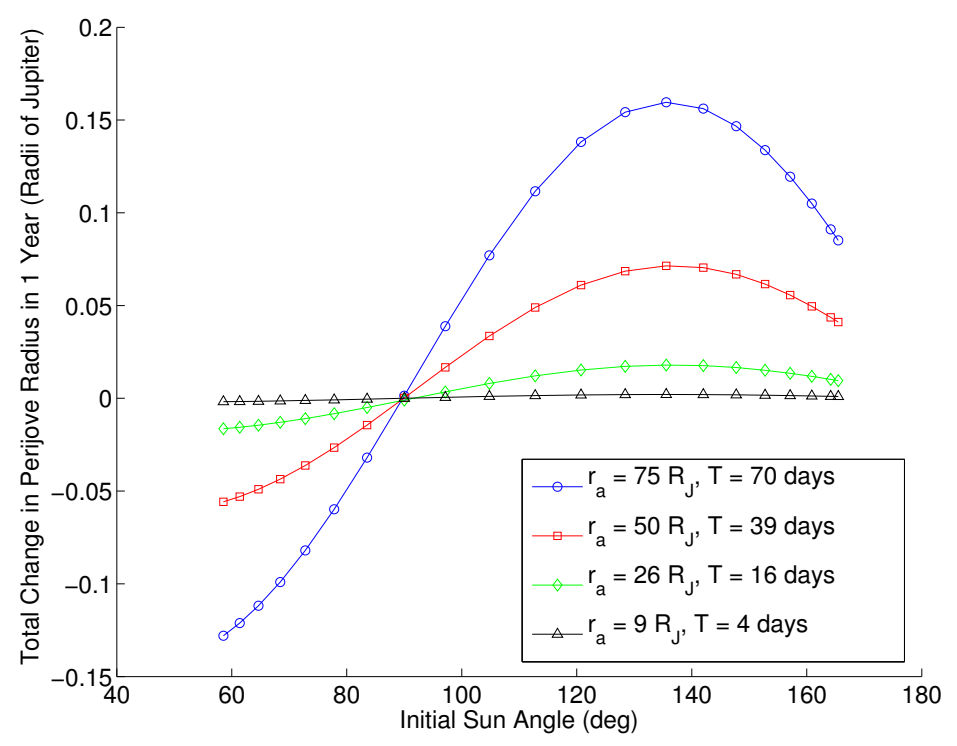

Figure 5.7: Change in perijove radius versus sun-line angle for various sized orbits.

It should also be noted that not only do these trajectories require a larger time of flight, but they also require a larger tether length to capture into the same sized orbit. This is due to the inherently higher $\mathrm{V}_{\infty}$ given by the non-Hohmann transfers, which require a larger force to become captured. 


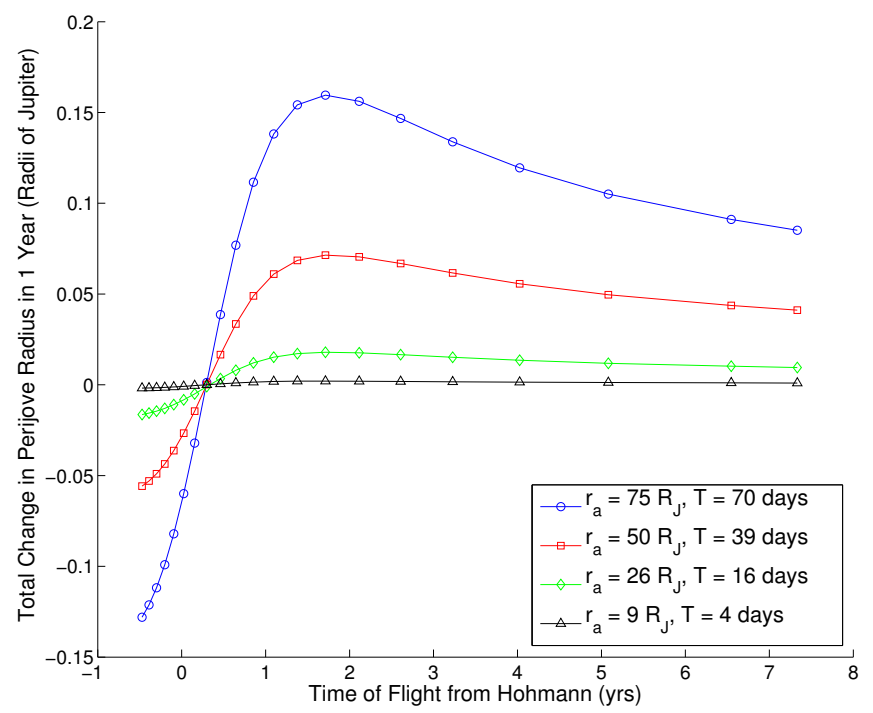

Figure 5.8: Change in perijove radius versus additional times of flight from a Hohmann transfer for various sized orbits.

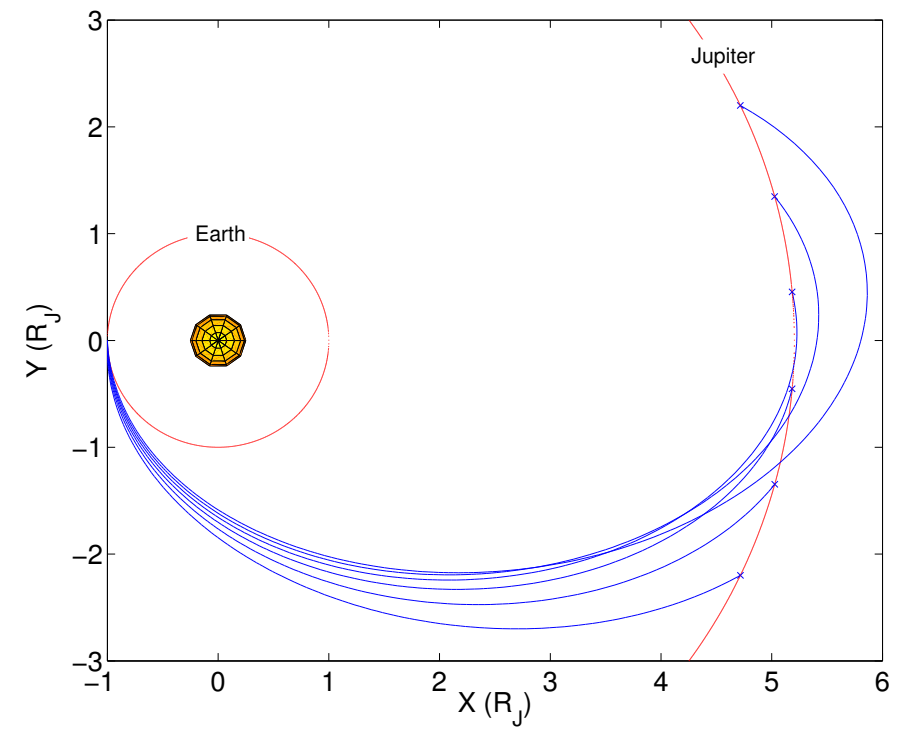

Figure 5.9: Lambert-targeted transfer orbits from Earth. 


\subsection{Ballistic Flybys}

The perijove pump-up phase can also be accomplished using flybys of the Galilean moons. This analysis is more complicated than the simplified phasefree case presented for the capture and apojove lowering phases, as many more flybys are needed and as such cannot be done with a phase-free assumption. An example of such a trajectory was found using EXPLORE, which is a grid-search trajectory optimization software developed at The University of Texas at Austin, and is shown in Figure 5.10.

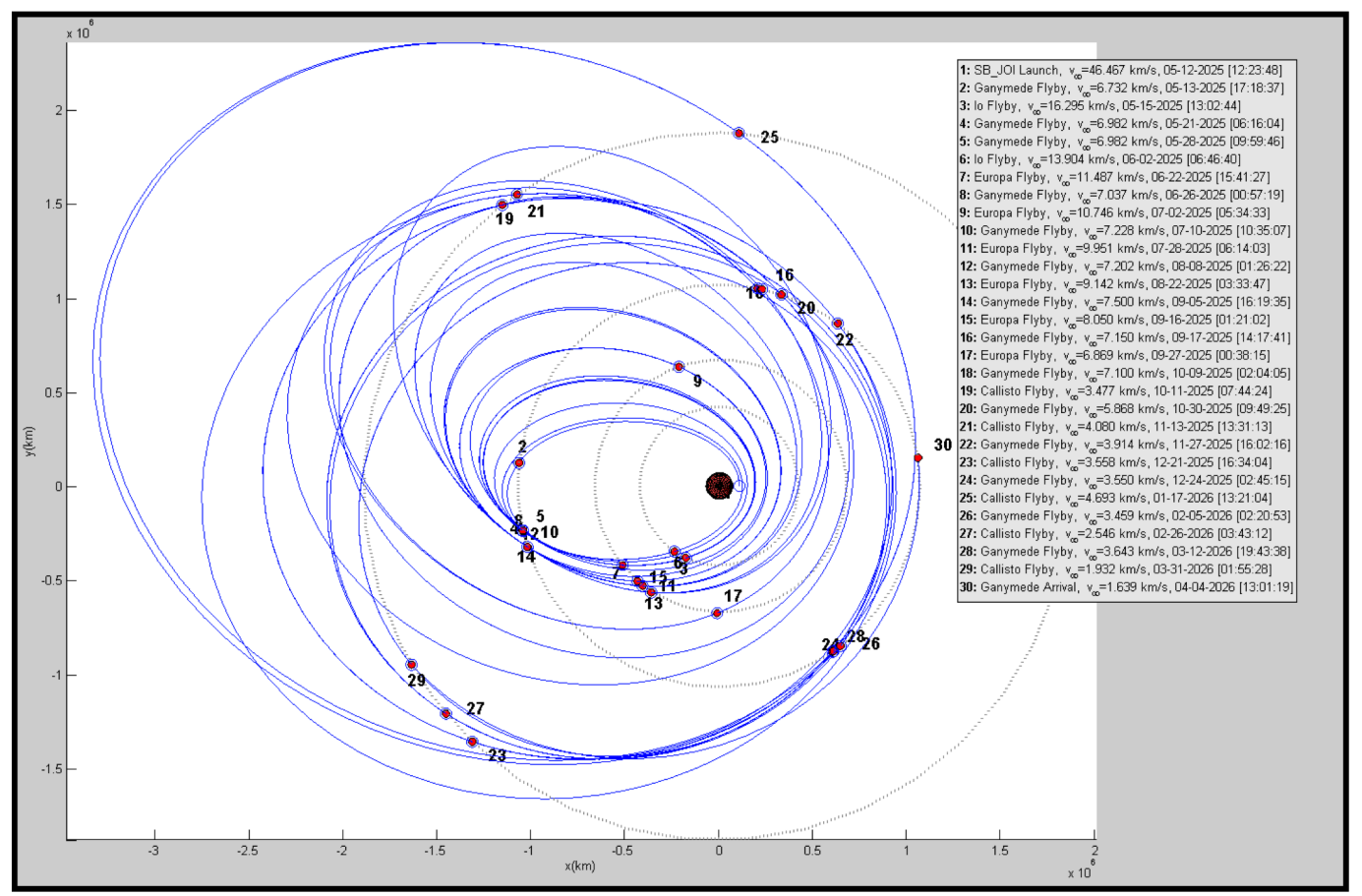

Figure 5.10: Ballistic trajectory using 28 flybys to raise perijove radius $\left(t_{f}=\right.$ 11 months). 
This perijove raising trajectory is a ballistic tour which uses 28 flybys and results in near Hohmann orbit between Callisto and Ganymede in just under a year. Other studies have accomplished this perijove raising maneuver using ballistic flybys on the similar time scale [10, 1], although these schemes required a sequence upwards of 20-30 flyby maneuvers. These types of trajectories are certainly viable options, although they are very complex and may not leave much room for error.

One possibility would be to use flybys in conjunction with the tether thrusting to raise the perijove radius, which is a topic for further study. The remaining option would be to utilize the secondary propulsion system, as mentioned previously, to raise perijove to the desired value. After the perijove of the orbit is raised to a certain value, the spacecraft may then begin placed into a loosely captured orbit with the desired moon [17]. 


\section{Chapter 6}

\section{Conclusions and Future Work}

The analysis conducted shows that electrodynamic tether propulsion could be a very useful and viable option for a mission to Jupiter. Such a propulsion system would eliminate many of the problems traditionally associated with such a mission, such as large amount of required propellent, or large mission durations if using a gravity-assisted trajectory.

\subsection{Summary}

Using a $50 \mathrm{~km}$ electrodynamic tether and minimum perijove radius of 2.0 $\mathrm{R}_{J}$, a $1000 \mathrm{~kg}$ spacecraft is shown to be captured and maneuvered into a pre-touring orbit (Ganymede-Europa Hohmann) in just under 2 years past entering Jupiter's sphere of influence. It is also shown that capture using a 20 $\mathrm{km}$ tether is feasible with the addition of at least one flyby of one of the larger Galilean moons and a perijove radius of $1.5 \mathrm{R}_{J}$. The maximum temperatures seen by these two spacecraft was shown to be within the expected range for such a close pass through a high radiation environment. These two trajectories were used as benchmarks for the later phases presented in the paper.

It is also shown that the tether alone is capable during the apojove pump-down phase, although the inclusion of flybys is helpful for both capture 
and lowering apojove only on the initial inbound hyperbola given a minimum tolerable perijove radius. Subsequent outbound only flybys are shown to be helpful in decreasing eccentricity, however in this scheme the perijove must kept constant. This can be accomplished by either the application of $\Delta \mathrm{V}$ burns via a secondary propulsion system at each apojove pass, the leveraging of solar perturbations, Lorentz forces via the application of a large active current through the tether, or some combination of these methods.

The remaining phase, before becoming loosely-captured by a moon, involves perijove pump-up maneuvers. Traditionally accomplished via $\mathrm{V}_{\infty}$ leveraging maneuvers [18], it is shown that this can be done purely utilizing the Lorentz force generated by the electrodynamic tether. It is shown that the tether is capable of accomplishing targeting a Europa-Ganymede Hohmann orbit in roughly 1.3 years of maneuvering. This analysis was done using a heuristic control law, and is not necessarily the optimal value. The addition of the net effects in perijove increase due to solar perturbations could shorten the required pump-up phase time, although the benefit of this is outweighed by the extra initial times of flight. Lastly, ballistic tours are examined as an alternative for perijove raising. Some combination of flybys and tether thrusting is assumed to be the ideal solution to this problem.

\subsection{Future Work}

As previously mentioned, some areas for future study include developing optimal tether control laws which incorporate realistic attitude dynamics of the tether. The structural dynamics (ie. bowing, tensile stresses, etc) of the 
tether are also not trivial issues, as are the radiation dosage accumulated by the spacecraft. The radiation may serve to be the main driving constraint for the capture trajectory. The power generation capabilities of electrodynamic tethers was briefly mentioned but remains a large topic of research in itself. Lastly, optimizing the perijove raising phase to use a combination of thrust from the tether and flybys remains an interesting and unsolved optimization problem.

The electrodynamic tether remains a promising option, especially in the case of capture and eccentricity reduction in systems with large external magnetic fields. This work can also be extended to the other planetary systems similar to Jupiter, such as Saturn and Uranus. 


\section{Bibliography}

[1] Lantoine, G. and et al., "MAGNETOUR: Surfing Planetary Systems on Electromagnetic and Multi-Body Gravity Fields," NASA Innovative Advanced Concepts (NIAC) Final Report, July 2013, pp. 1-103.

[2] Greeley, R. and Johnson, T., "The Jupiter Icy Moons Orbiter Project: The Scientific Rationale," EOS, Trans. Amer. Geophys. Union, Vol. 85, No. 36, Sept. 2004, pp. 337-343.

[3] Penzo, P. A., "A Survey of Tether Applications to Planetary Exploration," Adv. Astronaut. Sci., Vol. 62, 1986, pp. 71-88.

[4] Thompson, D. C. and et. al., "The Current-Voltage Characteristics of a Large Probe in Low Earth Orbit: TSS-1R Results," Geophys. Res. Lett., Vol. 25, No. 4, 1998, pp. 413-416.

[5] Katz, I. and et al., "Plasma Turbulence Enhanced Current Collection: Results from the Plasma Motor Generator Electrodynamic Tether Flight," Journal of Geophysical Research., Vol. 100, No. A2, Feb. 1995, pp. 16871690.

[6] Sanmartin, J. R. and et al., "Electrodynamic Tether at Jupiter - I: Capture Operation and Constraints," IEEE Trans. Plasma Sci., Vol. 21, No. 3, Oct. 2008, pp. 2450-2458. 
[7] Sanmartin, J. R. and et al., "Electrodynamic Tether at Jupiter - II: Fast Moon Tour After Capture," IEEE Trans. Plasma Sci., Vol. 37, No. 4, Apr. 2009, pp. 620-626.

[8] Sanmartin, J. R. and Lorenzini, E. C., "Exploration of Outer Planets Using Tethers for Power and Propulsion," Journal of Propulsion and Power, Vol. 21, No. 3, May-June 2005, pp. 571-576.

[9] Atchison, J. A. and Peck, M. A., "Lorentz-Augmented Jovian Orbit Insertion," Journal of Guidance, Control, and Dynamics, Vol. 32, No. 2, Mar. 2009, pp. 418-423.

[10] Campagnola, S. and Kawakatsu, Y., "Jupiter Magnetospheric Orbiter: Trajectory Design in the Jovian System," Journal of Spacecraft and Rockets, Vol. 49, No. 2, April 2012, pp. 318-324.

[11] Zanutto, D., Curreli, D., and Lorenzini, E. C., "Stability of Electrodynamic Tethers in a Three-Body System," Journal of Guidance, Control, and Dynamics, Vol. 34, No. 5, Oct. 2011, pp. 1441-1456.

[12] Charro, M. and et al., "A Proposed Two-Stage Two-Tether Scientific Mission at Jupiter," IEEE Transactions on Plasma Science, Vol. 40, No. 2, Feb. 2012, pp. 274-281.

[13] Bokelmann, K. and Russell, R. P., "Lyapunov Orbits In The Jupiter System Using Electrodynamic Tethers," AAS/AIAA Astrodynamics Specialist Conference, Vol. AAS 13-899, Aug 2013. 
[14] Bokelmann, K. and Russell, R. P., "Connecting Halo Orbits with Low Altitude Science Orbits at Europa," AAS/AIAA Astrodynamics Specialist Conference, Vol. AAS 13-789, Aug 2013.

[15] Bombardelli, C. and et al., "Io Exploration With Electrodynamic Tethers," AIAA/AAS Astrodynamics Specialist Conference, Aug. 2008.

[16] Yam, C. and et al., "Saturn Impact Trajectories for Cassini End-ofMission," Journal of Spacecraft and Rockets, Vol. 46, No. 2, Apr. 2009, pp. 353-364.

[17] Russell, R. P. and Lam, T., "Designing Ephemeris Capture Trajectories at Europa Using Unstable Periodic Orbits," Journal of Guidance, Control, and Dynamics, Vol. 30, No. 2, April 2007, pp. 482-491.

[18] Russell, R. P., "Survey of Spacecraft Trajectory Design in Strongly Perturbed Environments," Journal of Guidance, Control, and Dynamics, Vol. 35, No. 3, June 2012, pp. 705-720. 